\title{
elF4A inhibition circumvents uncontrolled DNA replication mediated by 4E-BP1 loss in pancreatic cancer
}

\author{
David Müller, ${ }^{1,2}$ Sauyeun Shin, ${ }^{1,2}$ Théo Goullet de Rugy, ${ }^{1}$ Rémi Samain, ${ }^{1,2}$ Romain Baer, \\ Manon Strehaiano, ${ }^{1,2}$ Laia Masvidal-Sanz, ${ }^{3}$ Julie Guillermet-Guibert, ${ }^{1}$ Christine Jean, ${ }^{1,2}$ \\ Yoshinori Tsukumo, ${ }^{4}$ Nahum Sonenberg, ${ }^{5}$ Frédéric Marion, ${ }^{6}$ Nicolas Guilbaud, ${ }^{6}$ \\ Jean-Sébastien Hoffmann, ${ }^{1}$ Ola Larsson, ${ }^{3}$ Corinne Bousquet, ${ }^{1,2}$ Stéphane Pyronnet, ${ }^{1,2}$ \\ and Yvan Martineau ${ }^{1,2}$
}

'Cancer Research Center of Toulouse (CRCT), INSERM UMR 1037, University Toulouse III Paul Sabatier, ERL5294 CNRS, Toulouse, France. 'Equipe Labellisée Ligue Contre le Cancer and Laboratoire d'Excellence Toulouse Cancer (TOUCAN), Toulouse, France. ${ }^{3}$ Department of Oncology-Pathology, Science for Life Laboratory, Karolinska Institutet, Solna, Sweden ${ }^{4}$ Molecular Diagnostics Section, Division of Molecular Target and Gene Therapy Products, National Institute of Health Sciences, Tokyo, Japan. ${ }^{5}$ Department of Biochemistry and Goodman Cancer Research Centre, McGill University, Montreal, Quebec, Canada. ${ }^{6}$ Research and Development Center, Laboratoires Pierre Fabre, Toulouse, France.

Pancreatic ductal adenocarcinoma (PDAC) relies on hyperactivated protein synthesis. Consistently, human and mouse PDAC lose expression of the translational repressor and mTOR target 4E-BP1. Using genome-wide polysome profiling, we here explore mRNAs whose translational efficiencies depend on the mTOR/4E-BP1 axis in pancreatic cancer cells. We identified a functional enrichment for mRNAs encoding DNA replication and repair proteins, including RRM2 and CDC6. Consequently, 4E-BP1 depletion favors DNA repair and renders DNA replication insensitive to mTOR inhibitors, in correlation with a sustained protein expression of CDC6 and RRM2, which is inversely correlated with 4E-BP1 expression in PDAC patient samples. DNA damage and pancreatic lesions induced by an experimental pancreatitis model uncover that 4E-BP1/2-deleted mice display an increased acinar cell proliferation and a better recovery than WT animals. Targeting translation, independently of 4E-BP1 status, using elF4A RNA helicase inhibitors (silvestrol derivatives) selectively modulates translation and limits CDC6 expression and DNA replication, leading to reduced PDAC tumor growth. In summary, 4E-BP1 expression loss during PDAC development induces selective changes in translation of mRNA encoding DNA replication and repair protein. Importantly, targeting protein synthesis by elF4A inhibitors circumvents PDAC resistance to mTOR inhibition.

Conflict of interest: The authors have declared that no conflict of interest exists

Copyright: () 2019, American Society for Clinical Investigation.

Submitted: April 29, 2018 Accepted: September 20, 2019 Published: November 1, 2019

Reference information: /CI Insight. 2019;4(21):e121951.

https://doi.org/10.1172/jici.

insight.121951.

\section{Introduction}

Pancreatic ductal adenocarcinoma (PDAC) remains an incurable disease. Although important progress has been made in patient care and combination therapies, the 5-year survival rate has not significantly raised in 2 decades (1). PDAC is remarkably resistant to conventional therapeutic strategies including chemotherapy, radiotherapy, and immunotherapy $(2,3)$. Therefore, extensive "omics" approaches have been applied to characterize PDAC driver and passenger mutations, as well as mRNA, long noncoding RNA (lncRNA), miRNA $(4,5)$, and protein and epigenetic profiles. These studies led to the discovery of multiple activated pathways and ample compensatory signaling crosstalks, accounting for PDAC adaptability to the stromal environment and current therapies. However, the weakest point of pancreatic cancer remains to be identified, especially for improving patient therapeutic management.

Regulation of protein synthesis provides quick and efficient coordination of gene expression with environmental changes (6). Consistently, protein synthesis is a key mechanism governing gene expression (7) and a node where oncogenic signaling pathways converge. Moreover, translation initiation factors are frequently dysregulated in human malignancies and thereby lead to the acquisition of cancer-associated phenotypes $(8,9)$. Changes in the efficiency of mRNA translation can be global, by affecting most mRNAs in a similar manner, or selective, by targeting mRNAs transcribed from a set 
of genes (10). Translational efficiency is commonly regulated at the initiation step, leading to modulation of the number of associated ribosomes per mRNA molecule and, consequently, altered protein levels. Translation efficiency can be quantified using polysome-profiling, whereby mRNAs are stratified based on how many ribosomes they associate with, and a pool of efficiently translated mRNAs is quantified. Another approach consists in ribosome profiling that indirectly infers translational efficiency by quantification of ribosome-protected fragments, yet allows the discovery of an alternative open reading frame $(11,12)$. Several studies applying polysome or ribosome profiling have shown that tumor aggressiveness is driven not only by global changes in protein synthesis, but also by selective changes in translational efficiency. Such selective changes can be achieved by modulating the activity of the eIF4F initiation complex. The complex is composed of eIF4E, a cap-binding protein; eIF4A, an RNA helicase; and eIF4G, a large scaffolding protein that mediates recruitment of the 40S ribosome subunit. Consequently, the $4 \mathrm{E}-\mathrm{BPs}$, which prevent eIF4F complex formation, have been pointed out as the main regulators of mTOR-controlled translation $(8,13-15)$.

Efficient protein synthesis was recently shown to be essential for PDAC development (16, 17). Moreover, an Nrf2 (a redox master regulator induced by oncogenic Kras in pancreatic cancer cells) transcriptional program protects specific translational factors from oxidation, allowing polysome formation and efficient protein synthesis (16). Accordingly, we previously showed an early loss of expression of the translational repressor 4E-BP1 during mouse and human pancreatic carcinogenesis (18). This event probably reflects the acquisition of a proliferative advantage during tumorigenesis (19) and may contribute to the observed PDAC resistance to mTOR inhibitors (18). Importantly, modulation of eIF4F activity by inhibition of mTOR $(9,10,12)$, or by 4E-BP depletion in tumors from other tissues (20), suggests that loss of 4E-BP1 in PDAC will lead to selective changes in translational efficiency, which may underlie their cancer-associated functions. Thus, we hypothesized that pancreatic tumors might be characterized by $4 \mathrm{E}-\mathrm{BP} 1-\mathrm{dependent}$ alterations of the translatome, leading to acquisition of procancer phenotypes.

By defining the translatome of human pancreatic cancer cells that depends on the mTOR/4E-BP1 axis, we here show that this pathway tightly controls the translation of a subset of mRNAs from genes involved in the licensing of replication origin and maintenance of the deoxynucleoside triphosphate (dNTP) pool. 4E-BP1 expression loss, therefore, enables pancreatic cancer cells to maintain their replication capacity, despite mTOR inhibition. Importantly, eIF4A inhibitors circumvent PDAC cell resistance to mTOR inhibitors by blocking both translation and replication processes. These data reveal a translational mechanism whereby cancer cells sustain their replication in PDAC tumor.

\section{Results}

Polysome profiling reveals mTOR-dependent translatome in PDAC. To identify mRNAs translated in a 4E-BP1dependent manner in PDAC, we used MiaPaca-2 cells, which express more 4E-BP1 protein than other pancreatic cancer cells $(18,21)$. Although mTOR inhibition and subsequent 4E-BP1 activation was shown elsewhere to affect translation of mRNAs encoding vimentin, YB1, survivin, MMP9, MMP3, MCL1, and cyclin D1 $(9,13,15)$, we did not find such regulations using mTOR inhibitor PP242 in MiaPaca-2 cells, except for cyclin D1 (Figure 1A, quantified in Supplemental Figure 1A; supplemental material available online with this article; https://doi.org/10.1172/jci.insight.121951DS1). This suggests that PDAC has a distinct translatome. We therefore studied mTOR/4E-BP1-sensitive translation in order to identify this specific dysregulation occurring upon 4E-BP1 loss (Figure 1B). This was performed by isolating cytoplasmic and efficiently translated (heavy polysome-associated) mRNAs from MiaPaca-2 cells upon PP242-mediated mTOR inhibition (Figure 1C). PP242 was previously shown to strongly inhibit translational efficiency via mTOR inhibition and 4E-BP1 dephosphorylation, with limited impact on cytosolic mRNA levels (10). To identify bona fide alterations in translational efficiency, we used the anota software package (12), which integrates variations in total mRNA levels to modifications of heavy polysome-associated mRNA levels. Although we observed changes in cytosolic mRNA levels (Figure 1D), we focused on translationally suppressed mRNAs, which are most likely to be directly targeted by the mTOR/4E-BP axis (Supplemental Table 1). Among the 682 mRNAs translationally suppressed by PP242, those encoding proteins controlling cell cycle progression were enriched (19\%, Figure 1E), including cyclin D3, as previously reported $(10,13)$. Strikingly, DNA replication (18\%) and repair (27\%) were the most affected processes following mTOR inhibition. Since such regulation was not reported for prostate or breast cancer cells $(9,13)$, translational control of DNA replication appears to be a PDAC-specific signature. 


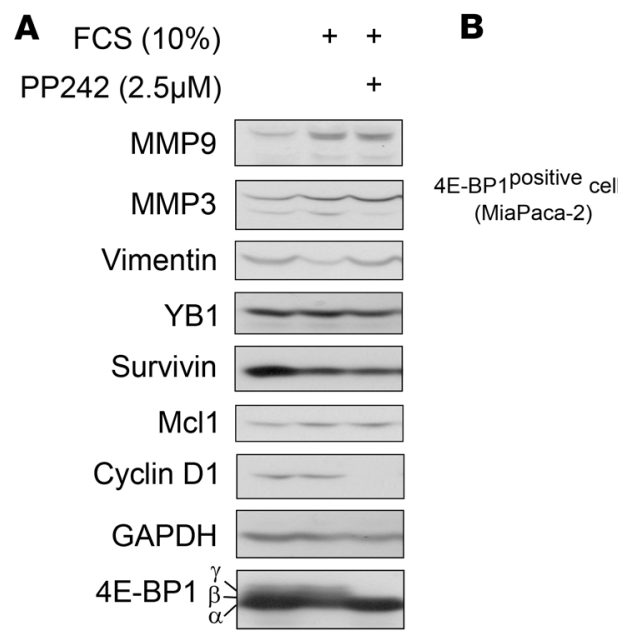

Protein
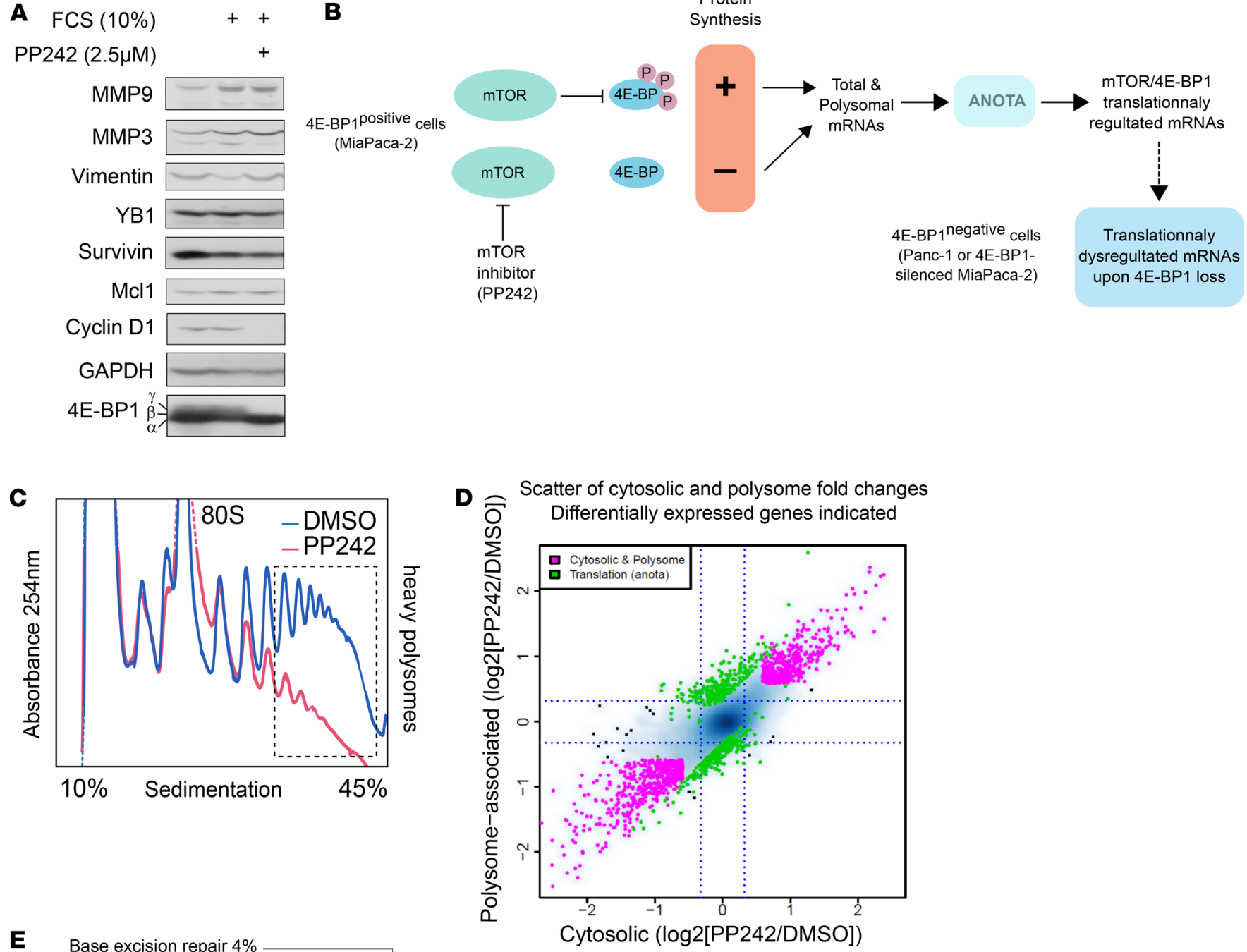

E Base excision repair 4\%

Pyrimidine metabolism 4\% Nucleotide excision repair $5 \%$ Homologous recombination $6 \%$ Mismatch repair $8 \%$

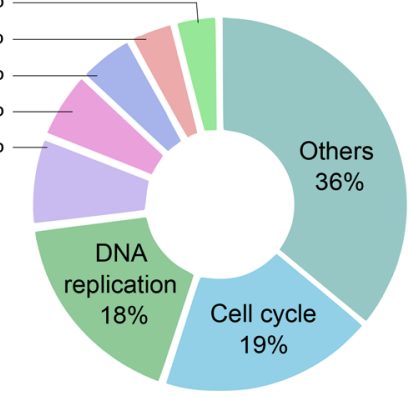

Figure 1. Polysome profiling allows the identification of the mTOR-dependent translatome in human pancreatic cancer cells. (A) Western blot analysis of indicated proteins in MiaPaca-2 cells. Cells were serum starved overnight and stimulated for 6 hours in the presence of $2.5 \mu \mathrm{M}$ PP242 or not with $10 \%$ serum (FCS). Respectively, $\alpha, \beta$, and $\gamma$ indicate hypo-, partially, and hyperphosphorylated forms of 4E-BP1 ( $n=3$ ). (B) Schematic of translatome analysis procedure. To identify translationally dysregulated mRNAs upon 4E-BP1 loss, we treated 4E-BP1-expressing (positive) MiaPaca-2 cells with mTOR inhibitor and purified total and polysomal mRNAs. The anota package allows the identification of mTOR/4E-BP1 translationally regulated mRNAs. Targets were then analyzed in 4E-BP1-negative cells. (C) Polysome profiles from MiaPaca-2 cells treated with DMSO or PP242 (0.5 $\mu \mathrm{M})$. Absorbance at $254 \mathrm{~nm}$ is shown as a function of sedimentation. Monosomes (80S) and heavy polysomes are indicated. (D) Graphical plot expressing changes in cytoplasmic and polysomal mRNA levels upon PP242 treatment. Genes showing modifications in both cytoplasmic and polysomal levels (pink) or only in polysomal levels (green) are indicated. (E) A pie chart of cellular functions of encoded proteins whose translation was suppressed by PP242. 
4E-BP1 mediates translational suppression of DNA replication genes in pancreatic cancer cells following $m T O R$ inhibition. To explore the importance of $4 \mathrm{E}-\mathrm{BP} 1$ in the translatome results, we analyzed translational control of identified mRNAs encoding proteins involved in DNA replication, including RRM2, CDC7, and CDC6. RRM2 plays a central role in deoxyribonucleotides synthesis, allowing maintenance of the dNTP pool, essential for DNA replication (22). CDC6 is a key component of prereplicative complexes, allowing the recruitment of MCM2-7 DNA helicases at the origins of replication (22). To mimic the loss of 4E-BP1 occurring in PDAC development, we silenced 4E-BP1 expression in MiaPaca-2 cells. We then analyzed the distribution of RRM2 and CDC6 transcripts across the polysome-profile following treatment with PP242 in shScr and sh4E-BP1 MiaPaca-2 cells. Upon PP242-mediated inhibition of mTOR, shScr MiaPaca-2 showed a marked inhibition of polysome formation as compared with sh4E-BP1 cells (Figure 2A). Quantitative PCR (qPCR) experiments showed that RRM2 and CDC6 mRNA were located in heavy polysomes in both cell lines, indicating their efficient translation. Upon mTOR inhibition, RRM2 and CDC6 mRNAs were less efficiently translated, as these shifted to lighter polysomes in shScr cells, while sh4E-BP1 cells showed no change in RRM2 and CDC6 mRNA distribution across the polysome-profile (Figure 2B). As a control, HPRT mRNA distribution did not significantly change in any of these cell lines. We analyzed RRM2 and CDC6 protein expression under the same conditions and found a significant reduction of both proteins' abundance when 4E-BP1 was dephosphorylated following mTOR inhibition. In contrast, in sh4E-BP1 cells, RRM2 and CDC6 proteins expression remained unchanged (Supplemental Figure 1B). Similar results were obtained using a second mTOR kinase inhibitor, Torin1, where CDC6 and RRM2 expression were maintained in the absence of 4E-BP1 (Figure 2C, quantified in Supplemental Figure 1C). As these effectors related to DNA replication and origin firing are synthesized in G1-phase, their protein expression levels were monitored in a synchronized cell population. shScr and sh4E-BP1 cells were treated with mTOR inhibitors following mitotic shake-off (Figure 2D, top and left). CDC6 and RRM2 expression was markedly reduced following PP242- and Torin1-mediated inhibition of mTOR in shScr Miapaca-2 cells (Figure 2D, right; quantified in Supplemental Figure 1D). Similarly, CDC7 protein, a key regulator of replication origin licensing, was downregulated (Figure 2D), which corresponded to its moderate shift to light polysomes (data not shown). In contrast, sh4E-BP1 cells showed a sustained expression of RRM2, CDC7, and CDC6, as in Figure 2C. Next, we overexpressed 4E-BP1 in Panc-1, which express low endogenous levels of 4E-BP1 protein (18, 21). Whereas RRM2 and CDC6 protein abundance remained constant upon Torin-1 treatment in Panc- 1 cells, lentivirus-mediated expression of 4E-BP1 reduced the amount of RRM2 and CDC6 under similar treatment (Supplemental Figure 1E). We compared CDC6 and RRM2 expression in response to mTOR inhibition in 4 pancreatic cancer cell lines, including AsPC-1 and Capan-2 (Supplemental Figure 1F). CDC6 expression was reduced by Torin 1 proportionally to 4E-BP1 expression, which is strongly downregulated in AsPC-1 and Capan-2. RRM2 expression was no longer reduced after Torin1 treatment in Panc-1, AsPC1, and Capan-2. Moreover, RRM2 abundance was globally increased in cells bearing weak expression of 4E-BP1 as compared with MiaPaca-2. We further confirmed the inverse relationship between 4E-BP1 loss and CDC6 or RRM2 expression using IHC in a series of 12 human PDAC samples. Consistent with in vitro data, 4E-BP1-negative PanIN lesions harbored strong RRM2 and CDC6 staining (Figure 2E), while 4E-BP1-positive PanIN lesions showed more variable CDC6 and RRM2 staining intensity. This variability in 4E-BP1-positive lesions could be explained by differences in 4E-BP1 phosphorylation, as a hyperphosphorylated 4E-BP1 would have a similar phenotype as 4E-BP1-negative PanIN. Altogether, downregulation of these targets corroborates polysome-profiling data and provides the first evidence to our knowledge of 4E-BP1 translational control of DNA replication and origin firing in pancreatic cancer cells.

$m$ TOR inhibition leads to DNA replication and repair inhibition via $4 E-B P 1$. We next assessed the effect of $4 \mathrm{E}-\mathrm{BP} 1$ loss on pancreatic cancer cell replication using quantitative image-based cytometry (QIBC). QIBC was applied to shScr and sh4E-BP1 MiaPaca-2 cells treated with mTOR inhibitors, in order to stimulate 4E-BP1 activity (Figure 3A). Upon PP242 treatment, the number of replicating shScr cells was significantly reduced after 3 hours, as attested by the decreased positivity to EdU (Figure 3B). sh4E-BP1 cells were far less susceptible, with minor changes in the number of EdU-labeled cells (Figure 3, A-C). Cell cycle monitoring also revealed a reduced replication activity compared with sh4E-BP1 cells, evidenced by a strong reduction in EdU intensity (Figure 3, A and C), which suggests a decrease in replicative DNA polymerase activity. Similar results were obtained with Torin1 (Supplemental Figure 1, G-I). Among the subset of genes implicated in the DNA replication process controlled by mTOR, some were also involved in DNA repair processes (such as RRM2, RPA1, and MCM family members). Thus, we assessed the effect of 4E-BP1 loss on DNA repair in pancreatic cancer cells. DNA damage and 
A

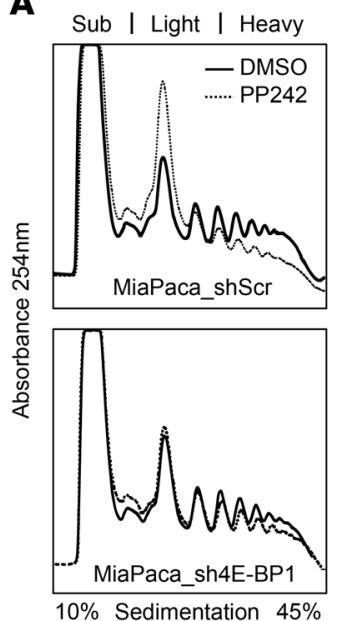

B

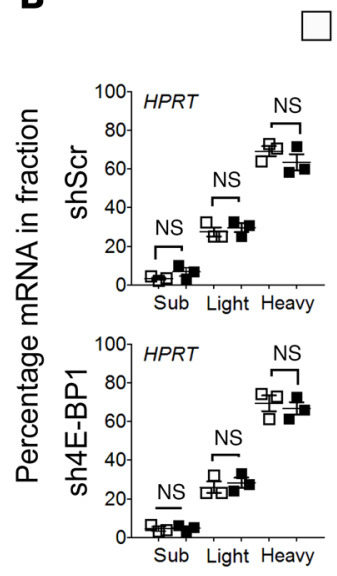

DMSO
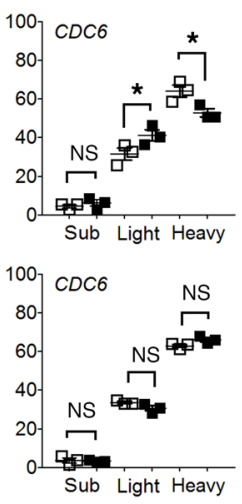

Fractions
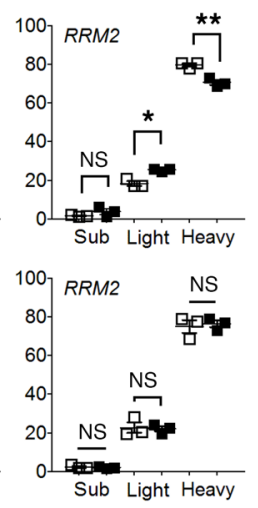

C

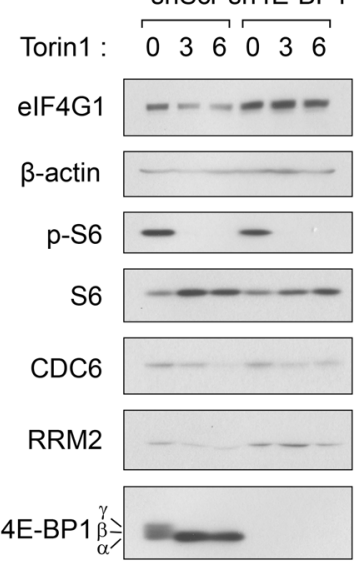

D
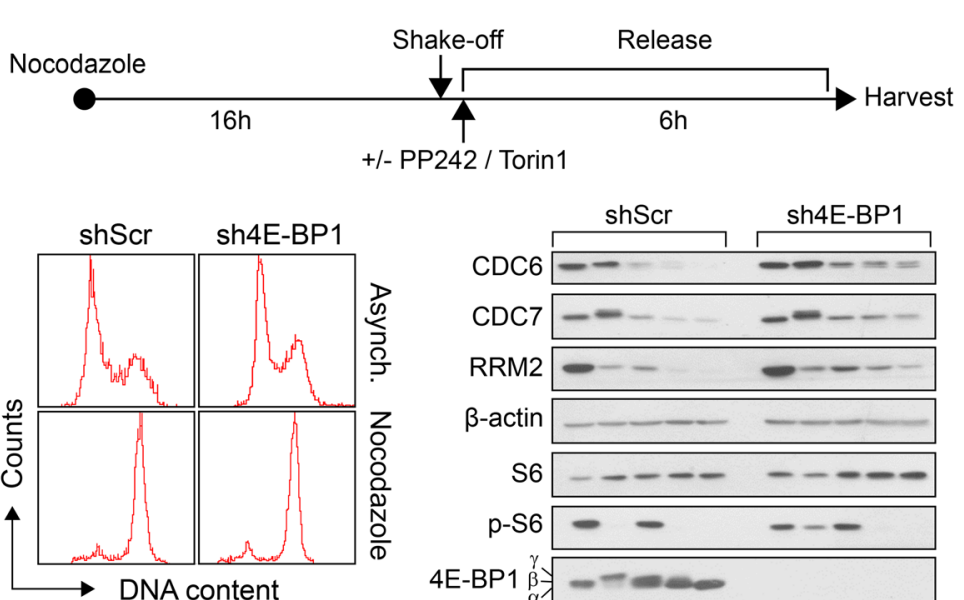

E
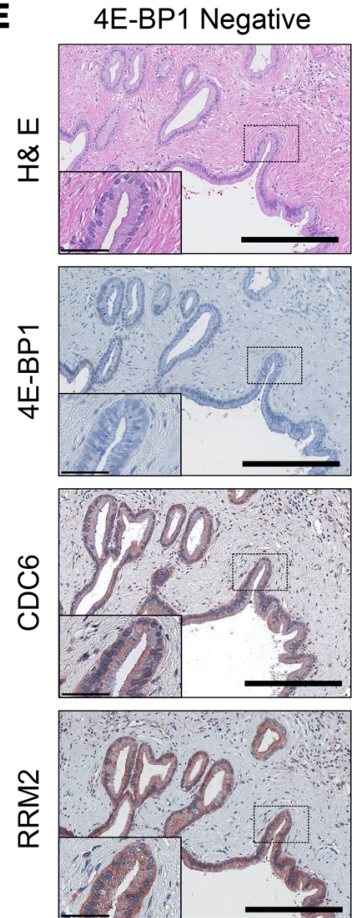

4E-BP1 Positive
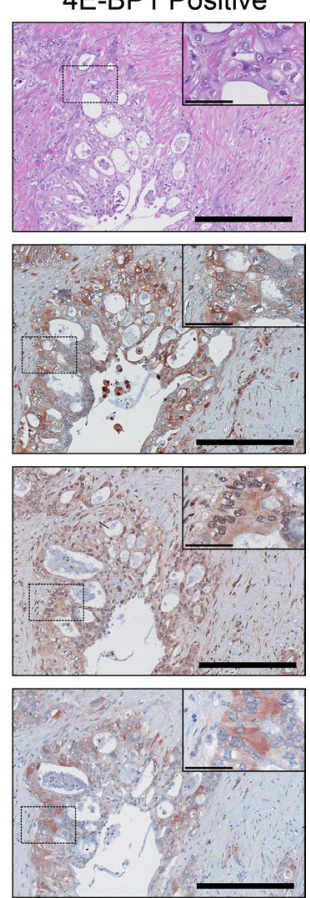

Figure 2. mTOR inhibition suppresses mRNA translation of genes involved in DNA replication via 4E-BP1. (A) Polysome profiles of MiaPaca-2 cells expressing (shScr) or not 4E-BP1 (sh4E-BP1) incubated with vehicle or $5 \mu \mathrm{M} \mathrm{PP242}$ for 3 hours. Absorbance at $254 \mathrm{~nm}$ is shown as a function of sedimentation. (B) The abundance of HPRT (control), RRM2, and CDC6 mRNAs along polysomes fractions was analyzed by real-time PCR. $P$ values were calculated using Student's $t$ test $\left({ }^{*} P<0.05 ;{ }^{* *} P<0.01\right)(n=3)$. (C) Western blot analysis of indicated proteins in shScr or sh4E-BP1 MiaPaca-2 cells treated with vehicle or Torin1 $(0.5 \mu \mathrm{M})$ for 3 hours and 6 hours. Respectively, $\alpha, \beta$, and $\gamma$ indicate hypo-, partially, and hyperphosphorylated forms of $4 \mathrm{E}-\mathrm{BP} 1$ ( $n=3$ ). (D) Nocodazole-arrested (Noco) shScr or sh4E-BP1 MiaPaca-2 cells were released for 6 hours and treated with either vehicle PP242 (0.5 $\mu$ M) or Torin1 (0.1 $\mu \mathrm{M}$ ). FACS profiles indicate the G2/M synchronization in both cell lines. Cell extracts were subjected to Western blot analysis. $\beta$-Actin served as a loading control ( 2 independent experiments). (E) Representative H\&E and IHC stainings of human 4E-BP1-negative (left) and 4E-BP1-positive (right) PanIN lesions from 12 patients. Scale bar: $500 \mu \mathrm{m}$. IHC using indicated antibody. Insets are higher magnification of cancer cells; 40x. Scale bar: $50 \mu \mathrm{m}$.

initiation of DNA repair are generally indicated by the formation of $\gamma \mathrm{H} 2 \mathrm{AX}$ and $\mathrm{p}-53 \mathrm{BP} 1$ foci in the nucleus (23). EdU labeling and QIBC analysis of MiaPaca-2 cells, following treatment with DNA damage-inducing agent mitomycin $\mathrm{C}$, was applied to quantify $\gamma \mathrm{H} 2 \mathrm{AX}$ (Figure 3D) and p-53BP1 foci (Figure 3E), specifically in replicating cells. Both markers' quantification showed that sh4E-BP1 Miapaca-2 cells were less susceptible to mitomycin $\mathrm{C}$-induced DNA damage and recovered faster from DNA damage. Together, these results suggest that 4E-BP1 loss in pancreatic cancer cells favors DNA replication and DNA repair. 
A
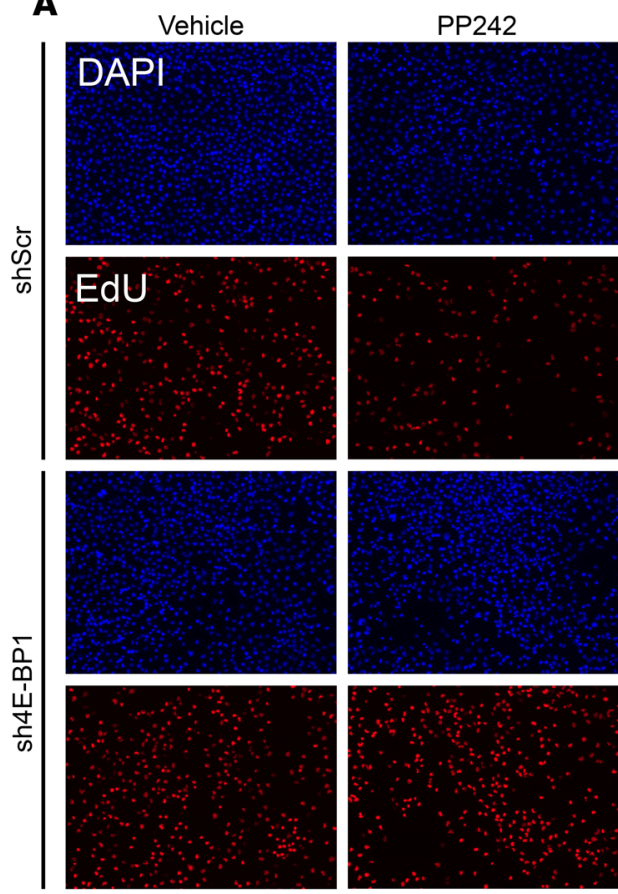
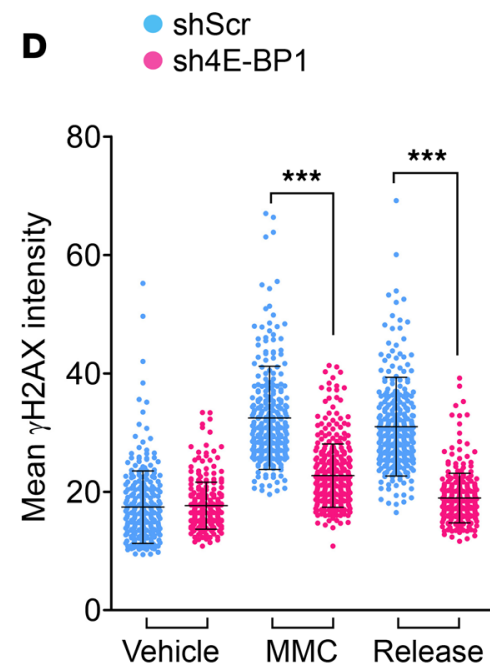

B
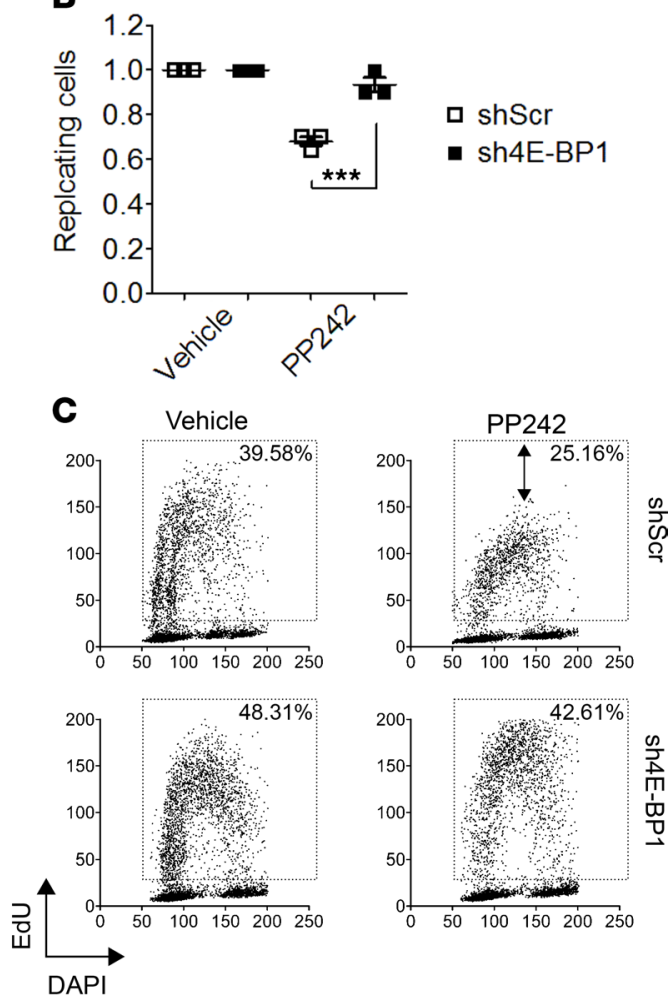

Figure 3. mTOR inhibition and consequent 4E-BP1 activation impedes DNA replication and DNA repair. (A) Asynchronous shScramble or sh4E-BP1 MiaPaca-2 cells were incubated with PP242 (5 $\mu \mathrm{M})$ for 3 hours and labeled with EdU. Nuclear DNA was counterstained by DAPI. (B) The proportions of replicating cells are shown as means and SDs and were generated from at least 3 independent experiments. $P$ values were calculated using 2 -way ANOVA (*** $P<$ 0.001). (C) Quantitative image-based cytometry (QIBC) single-cell analysis of EdU-labeled cells. Total EdU and DAPI values from $\mathbf{A}$ are plotted in a scatter diagram. Percentages indicate the proportion of replicating cells. Quantification of (D) $\gamma \mathrm{H} 2 \mathrm{AX}$-positive and (E) p-53BP1-positive cells in S-phase. Asynchronous cells were pulsed 30 minutes with EdU $(10 \mu \mathrm{M})$ prior to 2 hours mitomycin C treatment (MMC, $10 \mu \mathrm{g} / \mathrm{ml}$ ) and released for 4 hours. Mean $\gamma \mathrm{H} 2 \mathrm{AX}$ and p-53BP1 intensity was plotted for every EdU-positive cells in a scatter diagram. $P$ values were calculated using Student's $t$ test $\left.{ }^{* * *} P<0.001\right)$. Representative of 3 independent experiments.

Pancreatitis-induced DNA damage and recovery are favored in the absence of $4 E$-BPs. Induction of DNA damage in pancreatic tissue was recently described in acute pancreatitis, as visualized by $\gamma \mathrm{H} 2 \mathrm{AX}$ staining within acinar to ductal metaplasia (ADM) lesions (24). In mice, pancreatitis can be recapitulated by repeated i.p. injections of cerulein (Figure 4A) and is characterized by edema, ADM, and infiltration 

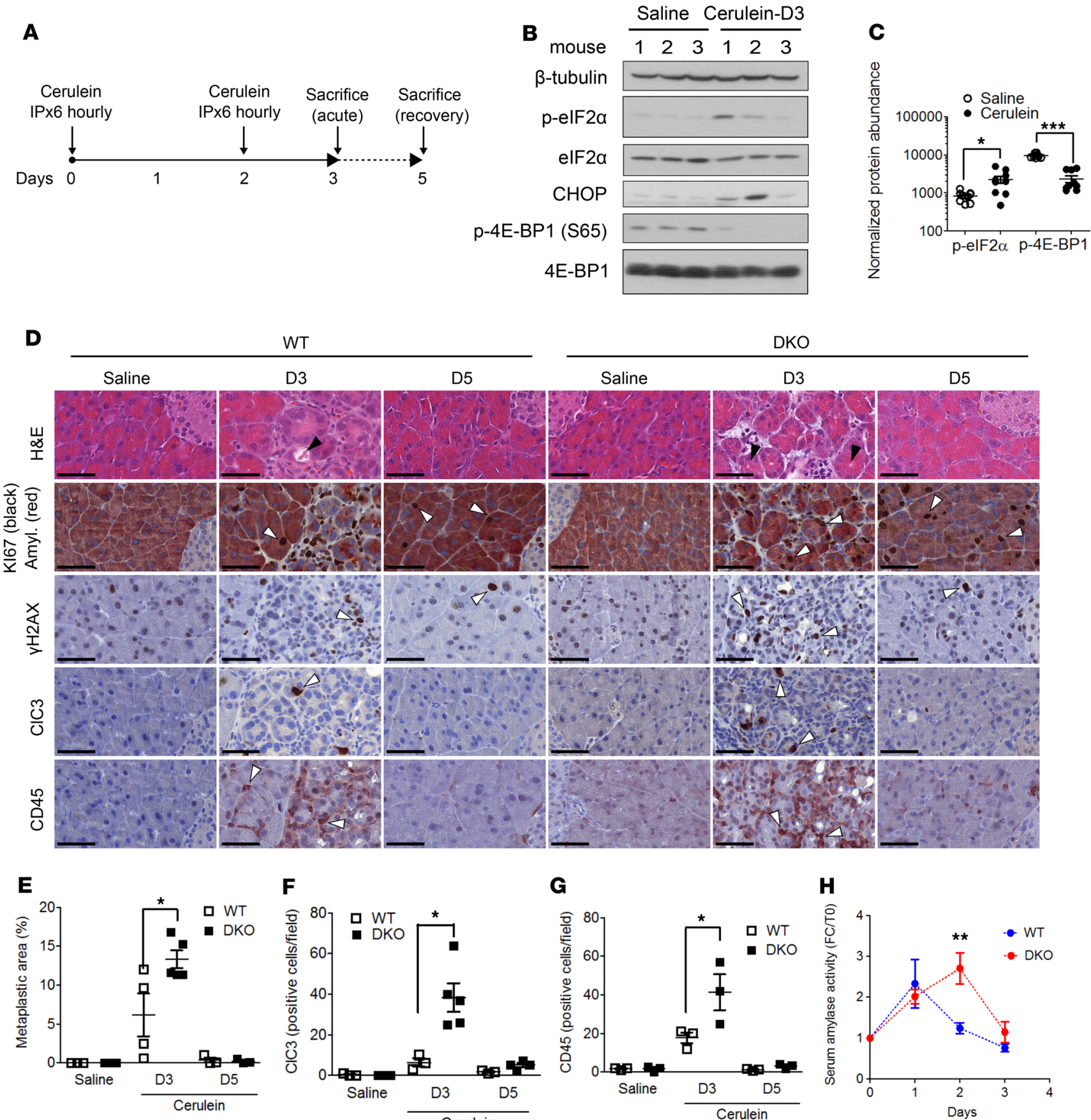

D3 $3-10$
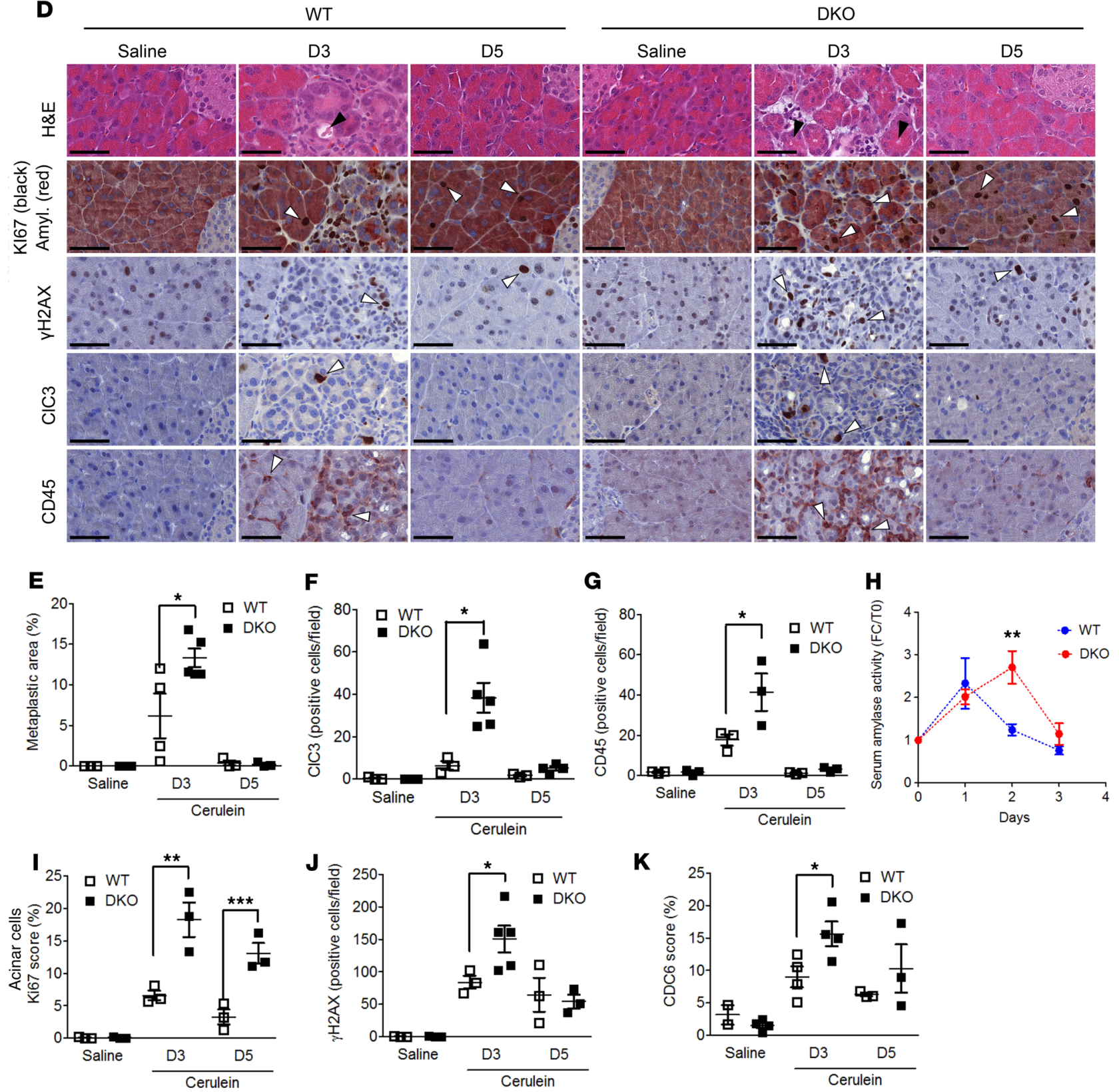

Figure 4. 4E-BP1 loss increases pancreatitis-induced metaplasia but favors recovery through enhanced proliferation and DNA repair. (A) Ceruleininduced pancreatitis experimental design. (B) Pancreatic extracts obtained from WT mice during acute phase (D3) were subjected to western blot analysis using indicated antibodies $(n=3)$. (C) Quantification of relative phosphorylated elF2 $\alpha$ and 4E-BP1 protein levels from B. $P$ values were calculated using Student's $t$ test $\left.{ }^{*} P<0.05,{ }^{* *} P<0.001\right)$. (D) Representative H\&E and IHC stainings of WT and 4E-BP1/2-KO (DKO) mice injected with cerulein during acute phase (D3) or after recovery (D5). Scale bar: $50 \mu \mathrm{m}$. IHC using indicated antibody. Black arrows indicate metaplastic lesions and interstitial 
expansion; white arrows indicate IHC staining. Representative of 2 independent experiments with 3-5 animals per conditions. (E) Quantification of ADM lesions/total pancreas surface. Quantification of (F) Cleaved-caspase 3-positive cells (CIC3) and (G) CD45-positive cells performed on 5 random $10 \times$ magnification fields in each mouse. (H) Measure of serum amylase during pancreatitis induction. Quantification of (I) Ki-67 positive acinar cells, (J) $\gamma \mathrm{H} 2 \mathrm{AX}$-positive cells, and (K) CDC6-positive cells performed on 5 random $10 \times$ magnification fields in each mouse. Mean \pm SEM. $P$ values were calculated using Student's $t$ test $\left({ }^{*} P<0.05 ;{ }^{* *} P<0.01 ;{ }^{* *} P<0.001\right)$.

of inflammatory cells, followed by a quick regeneration of pancreatic parenchyma via augmented cell proliferation. Cerulein was also reported to inhibit protein synthesis, in part through eIF $2 \alpha$ phosphorylation (25). We reproduced the latter observation together with the induction of the proapoptotic transcription factor $\mathrm{CHOP}$; we found that $4 \mathrm{E}-\mathrm{BP} 1$ was dephosphorylated and therefore activated, suggesting its potential role during acute pancreatitis (Figure 4, B and C). Based on the exacerbated cell cycle, DNA replication, and repair capacity of MiaPaca-2 cells lacking 4E-BP1 (Figure 3), we thought that the pancreas of mice lacking $4 \mathrm{E}-\mathrm{BPs}$ would have an enhanced ability to recover from acute pancreatitis. Thus, WT and 4E-BP1/2-double KO (DKO) mice - to avoid any potential compensation by 4E-BP2 - were subjected to cerulein injections followed by 2 days of recovery (Figure 4A). H\&E staining of injured pancreas indicated disordered acinar structures, abundant presence of metaplastic lesions, and interstitial expansion (Figure 4D, black arrows). 4E-BP1/2-DKO mice harbored an increased ADM formation and pancreatic damage, as attested by the total metaplastic area quantification (Figure 4E). This was associated with an increased positivity to anti-cleaved-caspase 3 (apoptosis) and anti-CD45 IHC stainings (inflammation) (Figure 4, D, F, and G). During the acute phase, serum amylase concentration was also increased in 4E-BP1/2-DKO animals (Figure 4H) as expected by 4E-BP's role in controlling acinar protein synthesis (25). Surprisingly, however, both genotypes showed complete histological recovery of pancreatic lesions at day 5 . We then assessed proliferation rate and DNA damage by anti-Ki67 and anti- $\gamma \mathrm{H} 2 \mathrm{AX}$ IHC stainings, respectively. Pancreata from 4E-BP-null animals showed a much higher proliferation rate of acinar cells at day 3 and 5, together with an enhanced $\gamma \mathrm{H} 2 \mathrm{AX}$ staining at day 3 (Figure 4, D, I, and J). In addition, CDC6 expression was enhanced in 4E-BP-null mice during pancreatitis and recovery phase (Figure $4 \mathrm{~K}$ ), in accordance with our previous results (Figure 2, C-E). These data indicate that mice lacking 4E-BPs are more susceptible to cerulein-induced pancreatitis, possibly through enhanced digestive enzyme secretion, although they completely recover from lesions, which is correlated to enhanced cell proliferation, DNA replication, and DNA repair.

Direct targeting of the eIF4F complex efficiently blocks $m R N A$ translation in a 4E-BP1-independent manner. Several therapeutic strategies have been developed to target the MAPK or PI3K/mTOR pathways, but only a few have been devoted to impeding eIF4F formation. However, elevated eIF4F activity is correlated with increased translation of mRNAs encoding oncogenic processes $(8,14)$. In order to bypass 4E-BP1 absence in PDAC and to provide an alternative to mTOR inhibition, we wished to directly target the translation initiation complex eIF4F. We used EC143.29 and EC143.69, two silvestrol analogs recently synthesized by Laboratoires Pierre Fabre (26). Silvestrol inhibits eIF4A RNA helicase activity, inducing a strong decrease of protein synthesis in cells. In vivo, silvestrol was shown to be a potent antitumor compound with moderate toxicity as a single agent $(27,28)$. First, we measured viability of shScr and sh4E-BP1 MiaPaca-2 cells treated with PP242, EC143.29, or EC143.69. Despite a much higher resistance to PP242 (one log of magnitude), sh4E-BP1 cells showed similar sensitivity to EC143.29 and EC143.69, as compared with shScr cells (Figure 5A). We then defined EC143.29, EC143.69, and PP242 IC50 on protein synthesis by coupling SUnSET (puromycin-based measurement of protein synthesis) and dot-blot approaches (Figure $5 \mathrm{~B})$. In shScr cells, the 3 compounds displayed a similar $\mathrm{IC}_{50}(\sim 10 \mathrm{nM})$, although PP242 did not induce a complete inhibition of protein synthesis even at high concentration (above $10 \mu \mathrm{M}$ ), as evidenced by the residual incorporation of puromycin (Figure 5C). This was confirmed by comparing polysome profiles from shScr cells treated with $\mathrm{IC}_{50}$ concentrations of EC143.29, EC143.69, and PP242, which show similar decreases in polysomal content (Figure 5D). More importantly, comparable decrease of heavy polysome fraction in shScr and sh4E-BP1 MiaPaca-2 cells treated with EC143.69 were observed (Figure 5E), as opposed to PP242, which alters polysome formation only in the presence of 4E-BP1 (Figure 2A). In addition, silvestrol derivatives affect neither Akt/mTOR signaling nor assembly of translation initiation factors at the cap structure (Figure 5, F and G), as described for other flavagline derivatives (29). Altogether, silvestrol analogs EC143.29 and EC143.69 show a high efficacy in blocking protein synthesis independently of 4E-BP1 expression. 
A
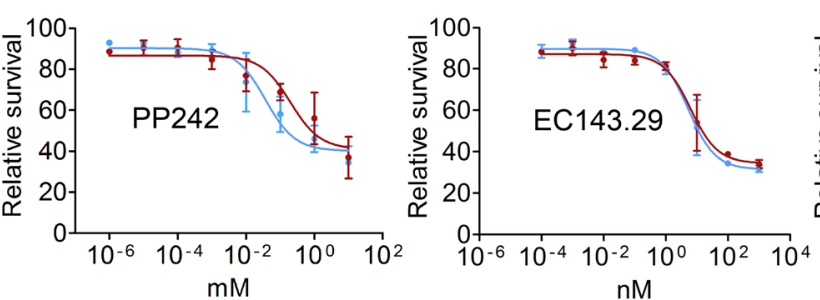

$\mathrm{nM}$

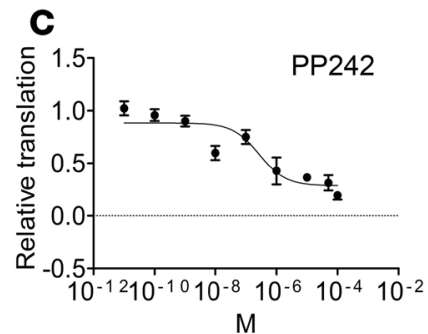

E
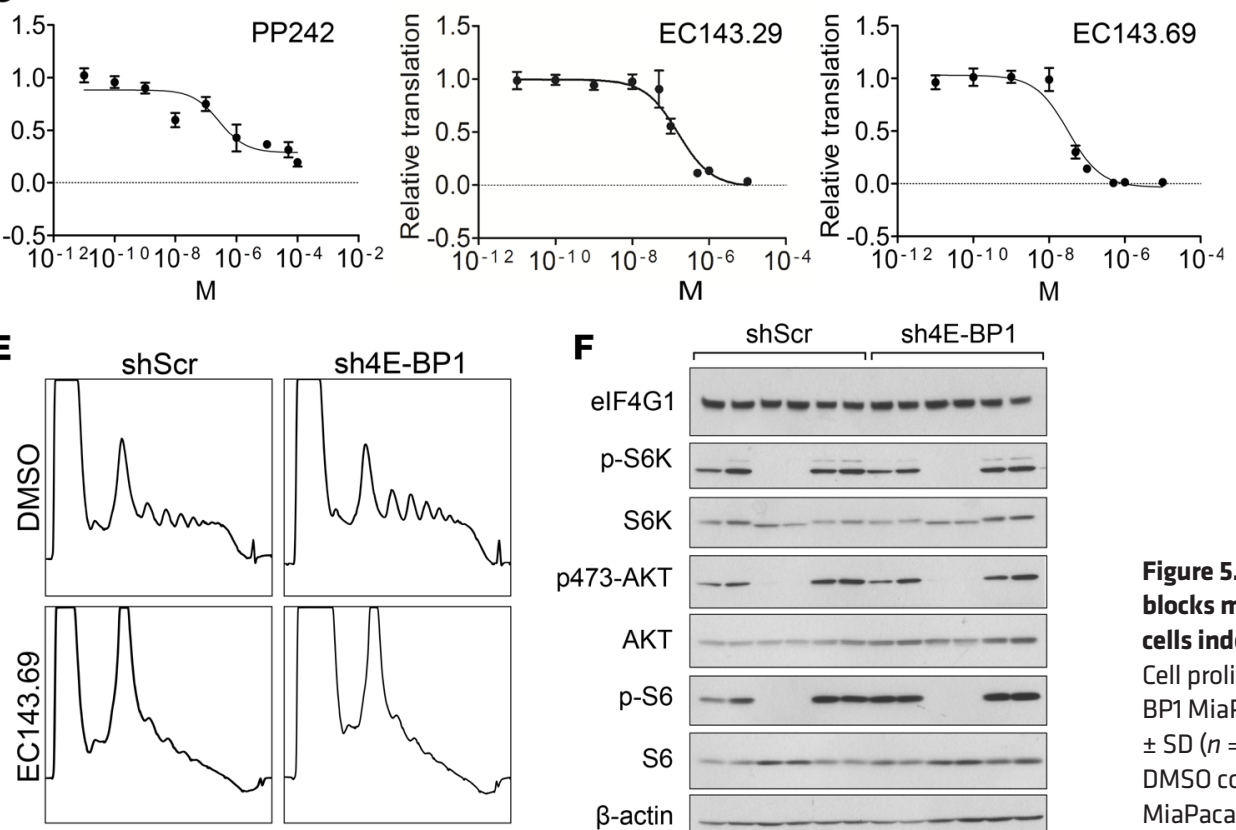

sh4E-BP1

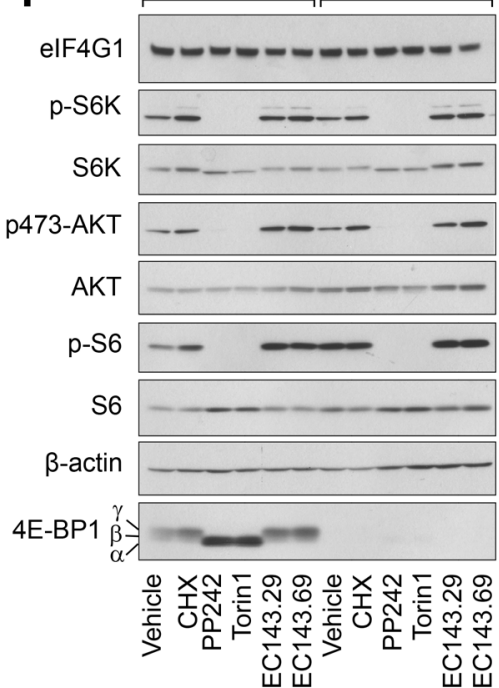

G
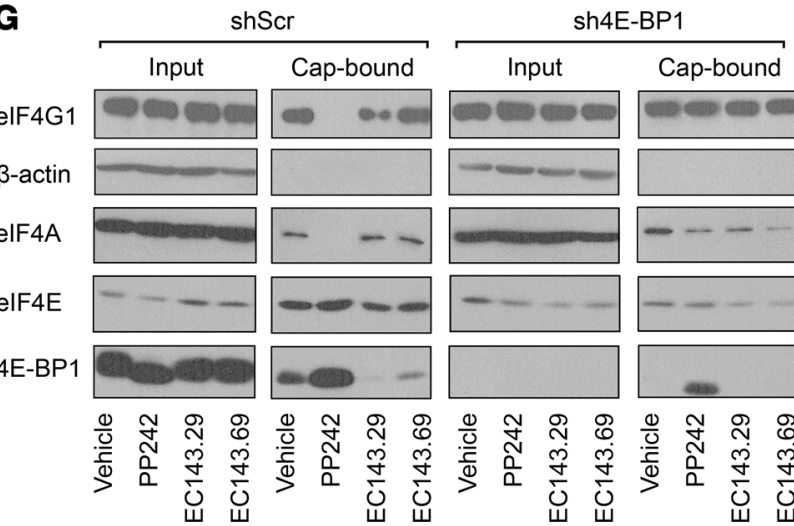
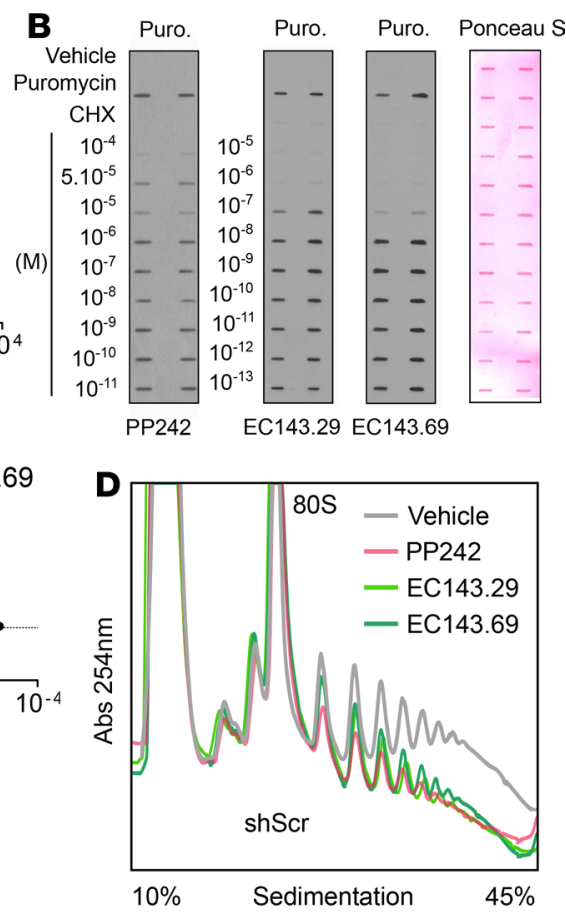

Figure 5. Targeting the elF4F complex efficiently blocks mRNA translation in pancreatic cancer cells independently of the mTOR/4E-BP1 axis. (A) Cell proliferation assays of shScramble or sh4EBP1 MiaPaca-2 cells. Data are presented as mean $\pm \mathrm{SD}(n=3)$. Survival is shown as compared with DMSO controls. (B and C) shScramble or sh4E-BP1 MiaPaca-2 cells were treated 1 hour with indicated drug concentrations. Representative dot blot result obtained from treated cells is depicted. (B) $\mathrm{CHX}$ is used as a negative control for puromycin incorporation. GAPDH is used as a loading control. (C) Representative curves of translation rate as a function of drug concentration (2 independent experiments). (D and $\mathbf{E})$ Polysome profiles of shScramble or sh4E-BP1 MiaPaca-2 cells incubated with vehicle, $2.5 \mu \mathrm{M}$ PP242, or 50 nM EC143.29 and EC143.69 for 3 hours. Absorbance at $254 \mathrm{~nm}$ is shown as a function of sedimentation $(n=2)$. (F) Western blot analysis of indicated proteins from whole cell lysate or (G) cap-bound complex from shScr or sh4E-BP1 MiaPaca-2 cells treated with vehicle or PP242 (2.5 $\mu M)$, Torin1 (500 nM), EC143.29 (50 nM), EC143.69 (50 $\mathrm{nM})$, cycloheximide (CHX, $100 \mu \mathrm{g} / \mathrm{ml})$ for 3 hours. Two independent experiments. Respectively, $\alpha, \beta$, and $\gamma$ indicate hypo-, partially, and hyperphosphorylated forms of 4E-BP1.

Silvestrol analogs abrogate DNA replication in pancreatic cancer cells and pancreatic tumor growth. We then assessed the impact of eIF4A inhibition on replication using QIBC. Upon EC143.69 treatment, the number of shScr and sh4E-BP1 replicating MiaPaca-2 cells was dramatically reduced (Figure 6 , $\mathrm{A}$ and $\mathrm{B}$ ). In addition, DNA replication was strongly diminished, as attested by the reduction of EdU intensity (Figure 6C). This was correlated with an important displacement of CDC6 mRNA from heavy to light polysomes, while $R R M 2$ mRNA remained actively translated (Figure 6D), as explained 
A

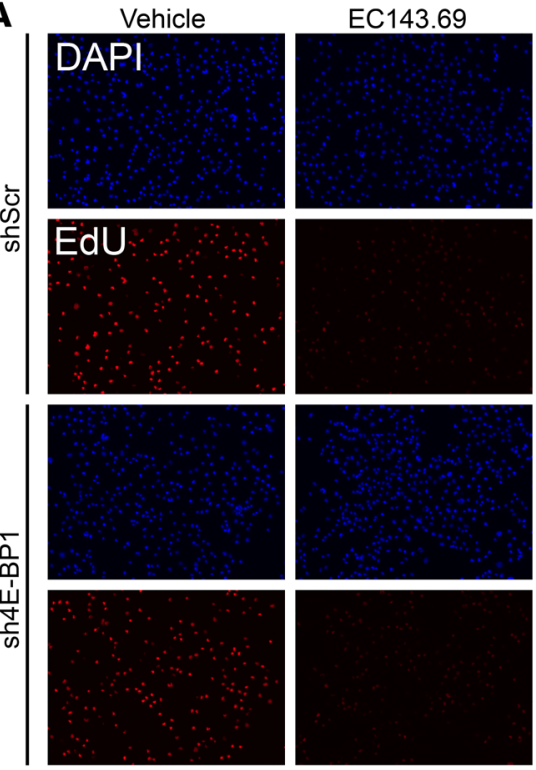

$\begin{array}{ll}\text { B } & \text { ahScr } \\ & \text { ah4E-BP1 }\end{array}$

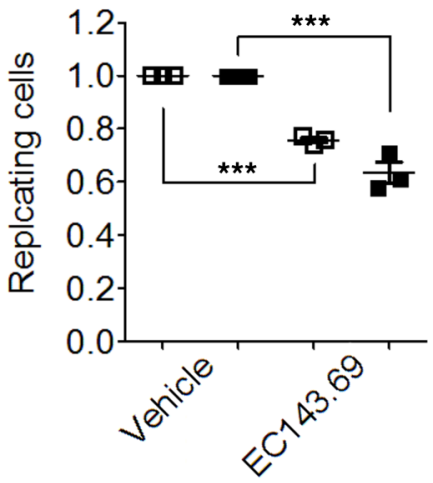

C
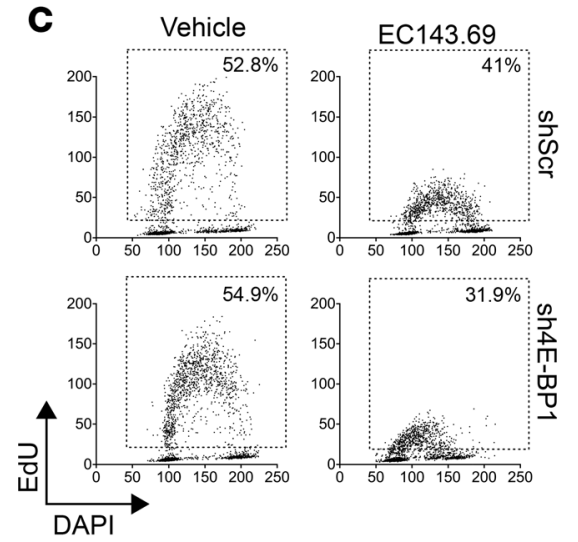

D
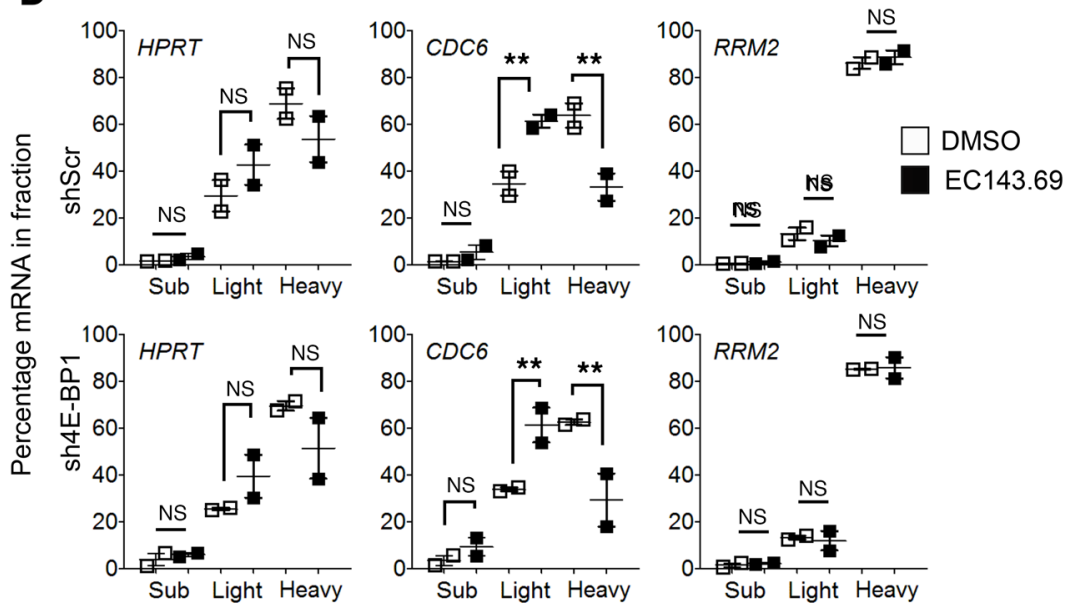

Fraction

E

elf

elF4G1

CDC6

RRM2

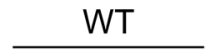

4E-BP1_KO

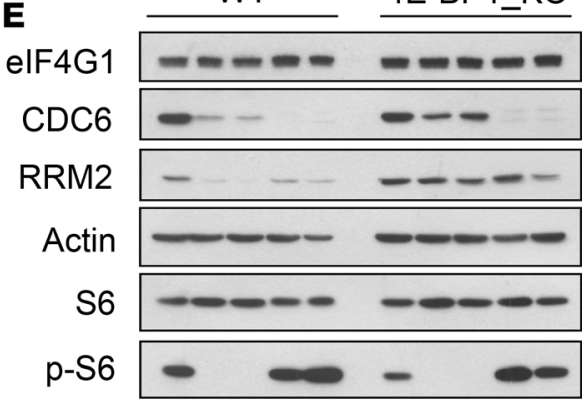

4E-BP1

4E-BP1

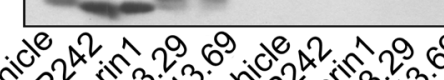

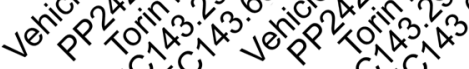

$\Leftrightarrow<0<0$

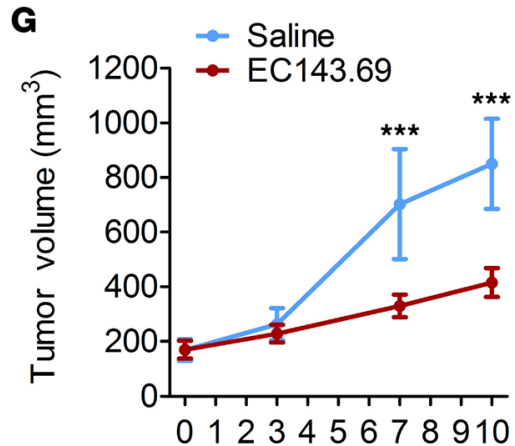

Days of treatment
$\mathbf{F}$

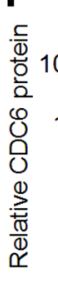
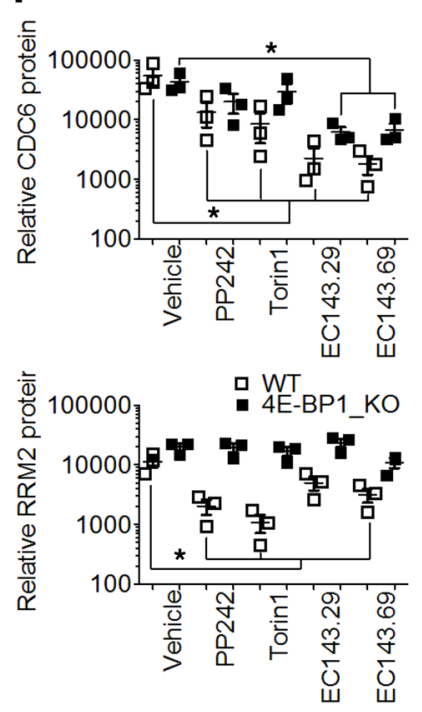

H

Vehicle

EC143.69

elF4GI

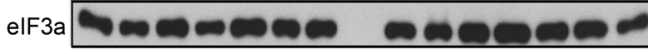

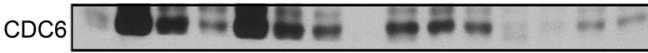

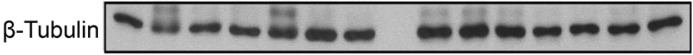

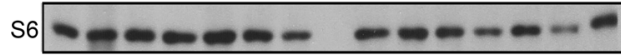

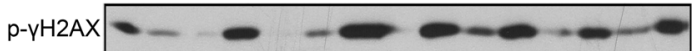

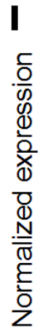

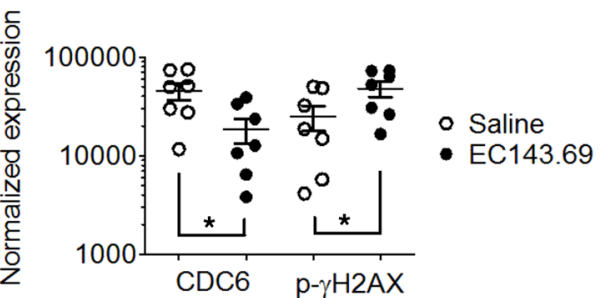

Figure 6. Silvestrol analogs abrogate DNA replication in pancreatic cancer cells and pancreatic tumor growth. (A) Asynchronous shScramble or sh4E-BP1 MiaPaca-2 cells were incubated with EC143.69 (50 nM) for 3 hours and labeled with EdU. Nuclear DNA was counterstained by DAPI. (B) The proportions of replicating cells are shown as means and SDs and were generated from at least 3 independent experiments. $P$ values were calculated using 2-way ANOVA (*** $P<0.001)$. (C) Quantitative image-based cytometry (QIBC) single-cell analysis of EdU-labeled cells. Total EdU and DAPI values from $\mathbf{B}$ are plotted in a scatter diagram. Percentages indicate the proportion of replicating cells. (D) The abundance of HPRT (control), RRM2, and 
CDC6 mRNAs along polysomes fractions was analyzed by real-time PCR. $P$ values were calculated using Student's $t$ test $\left({ }^{* *} P<0.01\right)$. Representative of 2 independent experiments. (E) Western blot analysis of indicated proteins in WT or 4E-BP1-KO MiaPaca-2 cells treated with vehicle or PP242 (2.5 $\mu \mathrm{M})$, Torin1 (500 nM), EC143.29 (50 nM), or EC143.69 (50 nM) for 3 hours. Representative of 3 independent experiments. (F) Quantification of relative CDC6 and RRM2 protein levels from E. $P$ values were calculated using Student's $t$ test $\left({ }^{*} P<0.05\right)$. (G) Syngeneic fibrotic pancreatic tumors were grafted s.c. in [57BI/6] mice. Mice were daily injected with EC143.69 or saline, and tumor growth was monitored at the indicated day. Seven animals per condition. Representative of 2 independent experiments. $P$ values were calculated using 2-way ANOVA (*** $P<0.001)$. (H) Western blot analysis of indicated proteins from tumor extracts in Saline-injected (left) or EC-143.69-injected (right) mice from G. (I) Quantification of relative CDC6 and p- $\gamma \mathrm{H} 2 \mathrm{AX}$ protein levels from $\mathbf{H}$. $P$ values were calculated using Student's $t$ test $\left({ }^{*} P<0.05\right)$.

by the preferential translational repression of mRNAs with long UTRs by eIF4A inhibitors (12). To rule out potential shRNA-mediated off-target effects, we recapitulated our findings using CRISPR/ Cas9-mediated EIF4EBP1 gene disruption in MiaPaca-2 cells. 4E-BP1-KO cells were insensitive to PP242-mediated replication arrest but remained sensitive to eIF4A inhibition (Supplemental Figure 2, $\mathrm{A}$ and $\mathrm{B}$ ). We further extended the analysis on the key regulators of DNA replication, RRM2 and CDC6, that were regulated by 4E-BP1. Upon treatment with EC143.29 and EC143.69, CDC6 expression was dramatically reduced in both WT and 4E-BP1-KO, as well as in shScr and sh4E-BP1 MiaPaca-2, cells. RRM2 amounts were barely affected by eIF4A inhibition in all cell lines (Figure 6, E and F, and Supplemental Figure 2C). Using another eIF4A inhibitor, RocA, we confirmed that global protein synthesis and CDC6 expression were reduced independently of 4E-BP1 levels, although RocA was less potent (Supplemental Figure 2C; $500 \mathrm{nM}$ as compared with $50 \mathrm{nM}$ for EC143 compounds). Using 4E-BP1-KO MiaPaca-2, mTOR inhibitor-mediated dowregulation of RRM2 and CDC6 was abolished (Figure 6, E and F, Torin1 and PP242 lanes). In contrast, cells expressing reduced 4E-BP1 levels (Panc-1 or sh4E-BP1 MiaPaca-2 cells) only show partial responses to mTOR inhibition (Supplemental Figure 1, B and F). This suggests that a minimal amount of 4E-BP1 is necessary to induce changes in RRM2 and CDC6 expression upon mTOR inhibition. Based on these observations, we questioned if $4 \mathrm{E}-\mathrm{BP} 2$ and $4 \mathrm{E}-\mathrm{BP} 3$ could have redundant functions with $4 \mathrm{E}-\mathrm{BP} 1$. Thus, CRISPR/ Cas9-mediated EIF4EBP2-KO and EIF4EBP3-KO MiaPaca-2 cells have been tested for their sensitivity to mTOR or eIF4A inhibition (Supplemental Figure 2D). We found that Torin1-mediated downregulation of CDC6 was observed independently of 4E-BP2 and 4E-BP3 expression, which confirm the key role of 4E-BP1 in MiaPaca-2. Consistently, EC143.69 induced a reduction of CDC6 expression in all genotype. In addition, using control or 4E-BP1-overexpressing Panc-1 cells, we showed that EC143.69-mediated eIF4A inhibition leads to a reduction of CDC6 protein independently of 4E-BP1 levels. In contrast, RRM2 and CDC6 expression were downregulated upon treatment with Torin1 only in 4E-BP1-overexpressing Panc-1 cells (Supplemental Figure 2E). Furthermore, eIF4A inhibition reduced CDC6 abundance in AsPC-1 and Capan-2 (Supplemental Figure 2F). In order to define which of eIF4A1 or eIF4A2 is responsible for the effect of silvestrol analogs EC143.29 and EC143.69, we performed siRNA-mediated depletion of eIF4A1 and eIF4A2 individually or simultaneously (Supplemental Figure 2G). CDC6 expression was diminished by depletion of eIF4A2, along with both 4A1 and 4A2 simultaneously, whereas RRM2 protein levels remained stable. As previously reported, eIFA2 expression was upregulated upon eIF4A1 silencing (30). Thus, CDC6 expression is mostly controlled by eIF4A2, suggesting that the latter is the predominant form of eIF4A expressed in MiaPaca- 2 cells.

Finally, we used a s.c. syngeneic fibrotic graft model of pancreatic tumor in C57B1/6J mice. This model presents a glandular organization of CK19-positive cancer cells surrounded by $\alpha$ SMA-positive fibroblastic cells (Supplemental Figure 3A) known to reduce chemotherapy efficacy (31). In addition, these tumors show histological similarities to LSL-Kras ${ }^{\mathrm{G} 12 \mathrm{D} /+} ; \mathrm{p}^{\mathrm{R}} 3^{\mathrm{R} 172 \mathrm{H} /+} ; \mathrm{Pdx} 1-\mathrm{Cre}$ mouse models (also referred as KPC) and human PDAC. Ten days after tumor implantation, mice were daily treated with EC143.69, and tumor growth was monitored. eIF4A inhibition reduced tumor development (Figure 6G and Supplemental Figure 3B) without impacting mouse body weight (Supplemental Figure 3C). Analysis of tumor extracts indicates that CDC6 expression was reduced upon EC143.69 treatment together with an increased $\gamma \mathrm{H} 2 \mathrm{AX}$ phosphorylation (Figure 6, H and I). Phosphorylated- $\gamma \mathrm{H} 2 \mathrm{AX}$ and cleaved-caspase 3 IHC analysis also revealed that eIF4A inhibition is associated with an increased replicative stress and apoptosis (Supplemental Figure 3, D and E). Altogether, the data demonstrate that silvestrol derivatives block pancreatic cancer cell proliferation by inhibiting DNA replication through inhibition of $C D C 6$ mRNA translation and reduce tumor growth in vivo. 


\section{Discussion}

Several reports have highlighted the crucial role of translational control along PDAC development $(16,17)$. We have previously shown the early loss of the key translational repressor 4E-BP1 in low-grade preneoplastic lesions of the pancreas (or PanIN), associated with an increased proliferation (18). Here, we aimed at uncovering the 4E-BP1-dependent translatome of human pancreatic cancer cells and highlighting a specific impact on DNA replication and repair processes. In contrast to many studies employing DNA microarrays and RNA sequencing, which have provided insights into the transcriptome of $\operatorname{PDAC}(4,5)$, our approach has addressed efficiently translated mRNAs, using polysome profiling. Translational control of DNA replication and repair appears to be a PDAC-specific signature, as these cellular processes were modestly impacted by translation inhibition in prostate and breast cancer cells $(2 \%-8 \%$ of translationally controlled genes) $(9,10)$.

Upon 4E-BP1 expression loss, the enhanced translation of mRNAs encoding DNA replication proteins, including our identified targets $C D C 6$ and $R R M 2$, should favor a sustained proliferation. Expression of RRM2, an essential component of the ribonucleoside diphosphate reductase complex, is critical to maintain the pool of dNTP during DNA replication. Thus, 4E-BP1-mediated downregulation of RRM2 would decrease the replicative DNA polymerases activity, as visualized by reduced EDU incorporation (Figure 3, A and B). Slowing down DNA replication could induce DNA replication arrest and consecutive appearance of stalled replication forks, a primer event to DNA double-strand breaks and genomic instability. Consequently, the sustained replication induced by 4E-BP1 depletion could explain the relative genomic stability of PDAC as compared with other cancers (32).

Clear illustrations of the essential role of protein synthesis and translational control in PDAC development have been reported. First, NRF2 has been shown to promote PDAC tumor maintenance by shielding components of the translation machinery from oxidation (16). Through this mechanism, NRF2 maintains a high level of protein synthesis and allows sustained proliferation of pancreatic cancer cells. In addition, phosphorylation of ribosomal protein S6 (rpS6), an essential event for the translation of mRNA containing a polypyrimidine tract, was shown to be required for PDAC development (17). Our finding now indicates that loss of 4E-BP1 in PDAC should favor protein synthesis and DNA replication. Importantly, this should be critical to sustain mRNA translation under conditions where mTOR activity is inhibited and 4E-BP1 is activated. These conditions include nutrient/energy deprivation, commonly found in PDAC tumor (2).

PDAC displays an impressive adaptability to therapeutic treatments (2). To circumvent PDAC resistance to mTOR inhibitors due to 4E-BP1 loss, we decided to target the only component of eIF4F complex harboring an enzymatic activity, the RNA helicase eIF4A. Mechanistically, 4E-BP1 and eIF4A have been described to regulate different subsets of mRNAs. mTOR/4E-BP1 can affect translation of mRNAs with short and long 5' UTRs whereas eIF4A mostly impacts translation of mRNAs with long UTRs (12). In accordance with this model, our translatome analysis revealed that $C D C 6$ and $R R M 2 \mathrm{mRNAs}$ were both sensitive to mTOR inhibition (Figure 2, B-D), while only CDC6 mRNA appeared to be impacted by eIF4A inhibition, since $C D C 6$ mRNA harbors a 212-nucleotide long 5' UTR, as opposed to RRM2 (60 nucleotides) (Figure 6, D-F, and Supplemental Figure 2, C-F). Silvestrol-sensitive transcripts were previously reported to contain G-quadruplex structures (33). However, analysis of CDC6 5' UTR using QGRS Mapper software (34) only identified 2 weak G-quadruplex structures (2 stacks). Our data rather support the model by which stem-loop structures and stability are more predictive to eIF4A inhibition (35), where CDC6 5' UTR is about 7 times more stable than RRM2 5' UTR, as measured using RNAfold ( $\Delta \mathrm{G}-89.29 \mathrm{Kcal} / \mathrm{mol} \mathrm{vs} .-12.9 \mathrm{Kcal} / \mathrm{mol}$, respectively).

Silvestrol derivatives are able to inhibit DNA replication and to block CDC6 expression, without impacting CDC7 or RRM2 protein levels. Along replication complex formation, CDC6 first recognizes ORC proteins, marking the DNA replication origins. Then, among many other factors, CDC7 and MCM27 are recruited to form the prereplication complex (22). Thus, targeting an early step of the replication complex formation (such as CDC6) appears sufficient to disable DNA replication (Figure 6, A-C, and E).

Among eIF4A inhibitors, flavaglines were described as potent single agents and to have synergistic effect with chemotherapy or targeted therapy on breast cancer, lymphomas, and melanoma mouse models (27, 28, 36). EC143.69 flavagline derivative from Laboratoires Pierre Fabre showed similar effects as a single agent on PDAC tumor growth, despite the marked drug resistance induced by PDAC stroma (31, 37). Resistance to eIF4A inhibitors has not been described, to our knowledge. In particular, the reported somatic mutations of EIF4A1 gene in cancers were sparse, distant from Phe-163 residue (shown to induce silvestrol resistance; ref. 29), and absent from PDAC samples (Cosmic database; ref. 38). As a consequence, eIF4A inhibitors arise as interesting companion molecules for chemotherapy in PDAC. This could be 
of particular interest if tumors expressed high levels of eIF4A2, as the latter appears essential to CDC6 expression (Supplemental Figure 2G).

To date, homoharringtonin/omacetaxine is the only drug that directly targets protein synthesis being approved for cancer treatment (8). Multiple clinical trials using inhibitors of upstream regulators of protein synthesis, such as dual PI3K-mTOR inhibitors or eIF4E kinase MNK, are ongoing (clinicaltrials.org). Among them, MNK1/MNK2 inhibitor EFT508 is currently tested on lymphoma. Moreover, previous reports have shown that the stress kinase MNK1 is activated and that eIF4E phosphorylation is massively increased upon pancreatitis (39), a major risk factor for PDAC development. eIF4E phosphorylation is also thought to drive translation of a specific subset of mRNAs $(40,41)$. In the absence of $4 \mathrm{E}-\mathrm{BPs}$, we previously showed that eIF4E is constitutively phosphorylated due to the stabilization of the eIF4E/eIF4G/ MNK complex (42). It is, thus, likely that enhanced eIF4E phosphorylation, consecutive to 4E-BP1 loss, contributes to the proliferative phenotype we describe (Figure 4, C and H). Finally, a potentially novel eIF4A inhibitor, eFT226, has been recently described and showed a great efficacy on B cell lymphoma in vitro and in xenograft model by inducing apoptosis (43). Here, we show similar efficacy of 2 silvestrol analogs, EC143.29 and EC143.69, on PDAC models by inducing DNA replication blockade. Converting these encouraging data on eIF4A inhibitors to a clinical setting constitutes a real challenge, where improvement of compound pharmacodynamics properties and tumor delivery are essential elements.

\section{Methods}

Cell lines and reagents. MiaPaca-2, Panc-1, AsPC-1, and Capan-2 cells were obtained from the ATCC and cultured in DMEM (MilliporeSigma) or RPMI (for AsPC-1) supplemented with 10\% FCS (Thermo Fisher Scientific) and $5 \mathrm{U} / \mathrm{ml}$ penicillin-streptomycin (Sigma-Aldrich) at $37^{\circ} \mathrm{C}$ and $5 \% \mathrm{CO}_{2}$. shRNA vector accession numbers are TRCN0000040203 for 4E-BP1 and SHC002 for Scramble (as described in ref. 18). Cells were seeded at $\sim 50 \%$ confluency, grown overnight, and treated using indicated concentrations of PP242 (MilliporeSigma), Torin1 (MilliporeSigma), Rocaglamide A (MilliporeSigma, SML0656), or EC143.29 (CAS Registry Number 185324735-4) and EC143.69 (CAS Registry Number 1853247-51-4), both provided by Laboratoires Pierre Fabre (26). As a control, cells were incubated in the presence of the vehicle (DMSO). Prior to mitotic shake-off, cells were treated with nocodazole $(0.1 \mu \mathrm{M}, 20$ hours) (Sigma-Aldrich) and released in fresh complete medium for 6 hours. DNA double-strand breaks were induced with Mitomycin C (MilliporeSigma; $10 \mu \mathrm{g} / \mathrm{ml}, 2$ hours) and released in fresh medium for 4 hours. WT, 4E-BP1-KO, 4E-BP2-KO, and 4E-BP3-KO Miapaca-2 cells were generated using CRISPR-Cas9 nickase system, as previously described (44). 4E-BP1-overexpressing Panc-1 cells (lenti 4E-BP1) and corresponding control cells (lenti Ctrl) were obtained after infection with pLenti CMV Blast HA-4E-BP1 and pLenti CMV Blast, respectively, followed by blasticidine selection at $4 \mu \mathrm{g} / \mathrm{ml}$ for 7 days.

Polysomal RNA isolation and microarray. Polysome profiling and $\mathrm{qPCR}$ were carried out as described (13). Briefly, cells were cultured in 15-cm dishes and treated with $2.5 \mu \mathrm{M}$ PP242 or vehicle (DMSO) for 12 hours. Cells were washed twice with cold PBS containing $100 \mu \mathrm{g} / \mathrm{ml}$ cycloheximide, collected, and lysed in a hypotonic lysis buffer (5 mM Tris- $\mathrm{HCl}$ [pH 7.5], $2.5 \mathrm{mM} \mathrm{MgCl} 2,1.5 \mathrm{mM} \mathrm{KCl}, 100 \mu \mathrm{g} / \mathrm{ml}$ cycloheximide, $2 \mathrm{mM}$ DTT, $0.5 \%$ Triton X-100, and $0.5 \%$ sodium deoxycholate). Lysates were loaded onto $10 \%-50 \%$ sucrose density gradients (20 mM HEPES-KOH [pH 7.6], $100 \mathrm{mM} \mathrm{KCl,} 5 \mathrm{mM} \mathrm{MgCl}$ ) and centrifuged at 217,000 $g$ (SW 40 Ti rotor, Beckman Coulter, Inc.) for 2 hours at $4^{\circ} \mathrm{C}$. Gradients were fractionated and the OD at $254 \mathrm{~nm}$ was continuously recorded using an ISCO fractionator (Teledyne ISCO). Polysome fractions with mRNA associated with more than 3 ribosomes were pooled (polysome-associated mRNA), and RNA was isolated using TRIzol (Thermo Fisher Scientific). A parallel sample was collected from the postnuclear lysates that were loaded onto the sucrose gradient (cytoplasmic mRNA), and RNA was isolated using TRIzol. For microarrays, 500 ng cytoplasmic or polysome-associated RNA ( $n=4$ from each condition) was used as starting material for the 3' IVT Express Kit (Affymetrix). The resulting labeled samples were probed with the Human Genome U133 Plus 2.0 gene arrays from Affymetrix according the instructions of the manufacturer and scanned using the GeneArray Scanner 3000. The microarray data reported in this paper have been deposited in the Gene Expression Omnibus (GEO) database under accession number GSE137553.

Polysome distribution RNA isolation and $q P C R$. Cells were washed, collected, and lysed in a hypotonic lysis buffer as previously described (18). Lysates were loaded onto $10 \%-45 \%$ sucrose density gradients $(20 \mathrm{mM}$ HEPES-KOH [pH 7.6], $100 \mathrm{mM} \mathrm{KCl,} 5 \mathrm{mM} \mathrm{MgCl}$ ) and centrifuged at 246,000 $\mathrm{g}$ (SW 55 Ti rotor, Beckman Coulter Inc.) for 45 minutes at $4^{\circ} \mathrm{C}$. Gradients were fractionated and the $\mathrm{OD}$ at $254 \mathrm{~nm}$ was continuously recorded using an ISCO fractionator. Polysome fractions were pooled 2 by 2 (polysome-associated mRNA), 
Table 1. qPCR primers

\begin{tabular}{|c|c|c|c|}
\hline Species & Transcript & Primer name & Sequence $\left(5^{\prime}-3^{\prime}\right)$ \\
\hline \multirow[t]{2}{*}{ Human } & HPRT & HPRTfw & GCTGAGGATTTGGAAAGGGTGT \\
\hline & & HPRTrv & ССАТСТССТTСАТСАСАТСТСС \\
\hline & & RRM2rv & GGCATCAGTCCTCGTTTCTT \\
\hline \multirow[t]{2}{*}{ Human } & CDC6 & CDC6fw & GCACAAСATTССTTCССТСTT \\
\hline & & CDC6rv & CCCACAGTGACCTCTTTCATTT \\
\hline
\end{tabular}

and RNA was isolated using TRIzol-LS (Thermo Fisher Scientific). A parallel sample was collected from postnuclear lysates that were loaded onto the sucrose gradient (cytoplasmic mRNA), and RNA was isolated using TRIzol. qPCR reactions were carried out using RevertAid H Minus Reverse Transcriptase (Thermo Fisher Scientific) and SsoFast EvaGreen Supermix (Bio-Rad) according to the manufacturers' instructions. The list of primers is provided in Table 1.

Proliferation analysis. Cell proliferation was measured using MTT reagent. MiaPaca- 2 cells were plated (5,000 cells per well) in 96-well tissue culture plates. After 24 hours, cells were treated with drugs or DMSO at indicated concentrations, in triplicate. MTT reagent was added to wells and incubated at $37^{\circ} \mathrm{C}$ for 2 hours after 48 hours of treatment. Formazan crystals were dissolved in $100 \mu \mathrm{DMSO}$. Plates were read at $450 \mathrm{~nm}$ on an Epoch microplate spectrophotometer (BioTek). Cell proliferation is expressed as a percentage of DMSO-treated cells.

SDS-PAGE, SUnSET, and Western blotting. Cells were harvested on ice, washed twice with cold PBS, and lysed in $50 \mathrm{mM}$ TRIS- $\mathrm{HCl}$ (pH 7.5), $150 \mathrm{mM} \mathrm{NaCl}, 1 \mathrm{mM}$ EDTA, and 1\% NP-40 supplemented with protease and phosphatase inhibitors (Roche). Protein concentration was measured using Protein Assay reagent (Bio-Rad), and equal amounts of proteins were subjected to SDS-PAGE and transferred onto nitrocellulose membrane (BioTraceNT; Pall Corp.). Western blots were quantified using ImageJ (NIH). For SUnSET assay, proteins were processed onto nitrocellulose membrane using HYBRI-SLOT Manifold. Membranes were washed in Tris Buffer Saline supplemented with $0.1 \%$ Tween 20 (TBS-T) and saturated in TBS-T with 5\% nonfat dry milk, incubated overnight with primary antibodies in TBS-T with 5\% BSA, washed, and revealed according to Cell Signaling Technology protocol. Dot blots were quantified using ImageJ. Drug concentrations were submitted to logarithmic transformation and quantification values to nonlinear regression using Graphpad Prism software. Results are expressed as log inhibitor vs. response.

Antibodies used in Western blot against MMP9 (catalog 255-1), YB1 (catalog 2397-1), Survivin (2 catalog 463), and Mcl1 (catalog 1239) were purchased from Epitomics; MMP3 (catalog D7F5B), Vimentin (catalog 5741), CyclinD1 (catalog 2978), 4E-BP1 (catalog 9644), eIF4G1 (catalog 8701), p-S6 (catalog 2215), S6K (catalog 2708), p-S6K (catalog 9234), eIF4A (catalog 2013), p473-Akt (catalog 2013), and Akt (catalog 9272) were purchased from Cell Signaling Technology; GAPDH (catalog 25778), S6 (catalog 74459), and CDC6 (catalog 9964) were purchased from Santa Cruz Biotechnology Inc., $\beta$-actin (catalog A5441) was purchased from MilliporeSigma; RRM2 (catalog 103193, lot no. 42718) was purchased from Genetex; CDC7 (catalog 108332), eIF4A1 (catalog 31217), and eIF4A2 (catalog 31218) were purchased

Table 2. Antibodies used for IHC

\begin{tabular}{clccc}
\hline Target & Source & Reference & Dilution & Antigen retrieval \\
4E-BP1 & Rabbit & CST 9644 & $1 / 500$ & Citrate \\
\hline CDC6 & Rabbit & Bethyl A302-486 & $1 / 500$ & Citrate \\
CD45 & Rabbit & Abcam 10558 & $1 / 300$ & Citrate \\
\hline Cleaved-caspase 3 & Rabbit & CST 9665 & $1 / 100$ & Citrate \\
\hline CK19 & Rabbit & Abcam ab52625 & $1 / 300$ & Tris-EDTA \\
\hline p- $\gamma$ H2AX & Rabbit & CST 9718 & $1 / 500$ & Tris-EDTA \\
Ki67 & Rabbit & Abcam ab16667 & $1 / 500$ & Tris-EDTA \\
\hline RRM2 & Rabbit & Cenetex 103193 lot 42718 & $1 / 500$ & Tris-EDTA \\
\hline$\alpha$ SMA & Rabbit & Abcam & $1 / 300$ & \\
\hline
\end{tabular}


from Abcam; and Puromycin 12D10 (catalog MABE343) was purchased from Merck Millipore. Rabbit (catalog 31460) and mouse (catalog 31430) secondary antibodies were form Pierce.

Immunofluorescence, microscopy and QIBC. Cells growing on 22-mm coverslips were fixed in formaldehyde 3.7\% (MilliporeSigma) for 10 minutes and permeabilized in Triton X-100 $0.1 \%$ for 10 minutes at room temperature. For EdU staining, cells were treated with $10 \mu \mathrm{M}$ EdU for 30 minutes before fixation and then stained using the Click-it Plus EdU Alexa Fluor 647 Imaging kit (Invitrogen) according to the manufacturer's instructions. Slides were mounted using fluorescent mounting medium (DAKO).

Images used in QIBC were obtained with an inverted wide-field microscope (Zeiss Axio Z1) equipped fast-switching filter wheels for excitation and emission of DAPI and Cy3 fluorescent dyes, as well as an Axiocam MRm CCD camera (Zeiss). NA $10 \times 0.4$ objective was used.

Acquisition times for the different channels were adjusted to obtain images in nonsaturating conditions and calibrated on the vehicle condition. Six images were acquired for each condition, containing in total 4,0006,000 cells per condition. After acquisition, images were processed for automated analysis using the CellProfiler software. DAPI signal was used for segmentation of the nuclei according to intensity threshold, generating a mask that identified each individual nucleus as an individual object. Data generated by CellProfiler were used to quantify the percentage of cells in each phase of the cycle. p-53BP1 (catalog 2675) antibody was obtained from Cell Signaling Technology, and $\gamma \mathrm{H} 2 \mathrm{AX}$ (Ser109, clone JBW301) antibody from Merck Millipore.

Cap ( $m 7 G T P)$ pull-down assay. Cap pull-down assay was carried out as described (13). Briefly, cells were lysed in the cap pull-down buffer (40 mM HEPES KOH [pH 7.5], $120 \mathrm{mM} \mathrm{NaCl}, 1 \mathrm{mM}$ EDTA, $0.1 \mathrm{mM}$ GDP, and $0.3 \%$ CHAPS) containing protease and phosphatase inhibitors. Protein extract (1 mg) was incubated for 2 hours at $4^{\circ} \mathrm{C}$ with m7GTP-agarose beads (Jena Bioscience). Beads were washed 3 times with the cap pull-down buffer and eluted by boiling in $2 \times$ Laemmli buffer for 5 minutes. m7GTP-bound proteins were visualized by Western blotting.

Cerulein-induced pancreatitis. WT and Eif4ebp1;Eif4ebp2-DKO mice were previously described and maintained in a C57B1/6J background (45). Ten- to 14-week-old mice were subjected to 2 series of 6 hourly i.p. injections of cerulein (Bachem AG) at a concentration of $75 \mu \mathrm{g} / \mathrm{kg}$ on alternating days separated by 24 hours. Saline was injected as control. Littermate controls (WT) were injected in parallel with the experimental animals (DKO). Serum amylase activity was measured using the Phadebas Amylase Test (Magle) according to the manufacturer's instructions.

SiRNA transfection. MiaPaca-2 cells were transfected with $20 \mathrm{nM}$ of Silencer Select siRNA targeting eIF4A1 (Ambion, s4567), eIF4A2 (s4572), or a nontargeting siRNA (SiGenome control, Dharmacon) using Lipofectamine 2000 transfection reagent (Invitrogen) according to the manufacturer's instructions.

Histology and immunostaining. Pancreatic tissues from both control (WT) and experimental mice (DKO) were fixed overnight in 10\% neutral buffered formalin, embedded in paraffin, and sectioned (4- $\mu \mathrm{m}$ thick). Histopathological features were determined using H\&E. Immunostaining was conducted using standard methods on formalin-fixed, paraffin-embedded tissues. Antigen retrieval and antibody dilution were carried out as described in Table 2. Human PDAC samples were previously described (18). One representative slide per mouse was imaged using a Hamamatsu Nanozoomer 2-slide scanner (Hamamatsu Photonics). ADM lesions were measured on the entire section with the Nanozoomer Digital Pathology view software (Hamamatsu). Quantification of nuclear staining was performed using Image $(\mathrm{NIH})$ with Color deconvolution plugin and a batch processing macro. Nonacinar Ki67 staining was manually excluded.

Syngeneic tumor graft. Mouse pancreatic tumor cells were isolated from the pancreas of KPC (37). A total of $40 \times 10^{3}$ tumor cells together with $12 \times 10^{4}$ activated mouse fibroblasts were s.c. injected in immunocompetent C57B1/6J mice to generate a fibrotic tumor similar to those observed in the KPC mouse model. When syngeneic graft reached $1 \mathrm{~cm}^{3}$, tumor was removed and cut into fragments (2-3 mm edge length). Fragments were s.c. implanted with a $14 \mathrm{G}$ biopsy needle (Bard Magnum) in the right upper flank of anesthetized C57Bl/6J mice $(n=14)$. Ten days after tumor implantation, mice were randomized in 2 groups with equivalent mean group tumor volumes (about $170 \mathrm{~mm}^{3}$ ). Mice were daily i.p. injected with EC143.69 at $2 \mathrm{mg} / \mathrm{kg}$ or with saline solution for 10 days. Tumor volume was monitored at days 3, 7, and 10 using a slide caliper and calculated using the following formula $\mathrm{V}=$ Length $\times \mathrm{Width}^{2} \times 0.5$. At sacrifice, tumors were split into 2 fragments, 1 for histology and IHC 1 one for Western blotting analysis, as described above.

Statistics. As indicated in the figure legends, statistical significance was determined using 2-way ANOVA or Student's $t$ test with 2 tailed $P$ value. All values are mean \pm SD. A $P$ value less 0.05 was considered statistically significant $\left({ }^{*} P<0.05 ;{ }^{* *} P<0.01 ; * * * P<0.001\right)$. 
Study approval. All experiments using animals were done in accordance with the principles and guidelines established by INSERM Anexplo UMS006 and were approved by the institutional and regional animal care and use committees.

\section{Author contributions}

DM, RB, SS, and YM designed and carried out experiments. RS, MS, and CJ performed experiments. LMS and OL analyzed microarrays data using anota. TGDR designed experiments. DM and YM conceived the study. SS and YM carried out experiments for revision. NS provided 4E-BP1/2-DKO mice. YT provided 4E-BP1-KO and 4E-BP2-KO MiaPaca-2 cell lines. MiaPaca-2 cell lines. FM and NG provided EC143.29 and EC143.69. SP and YM supervised the study. CB, SP, and YM provided funding. TGDR, JSH, JGG, and OL provided critical advice regarding the experimental design. DM and YM drafted the manuscript. JGG, JSH, NS, OL, CJ, CB, and SP edited the manuscript.

\section{Acknowledgments}

We thank FX Frenois from the Imag'IN Plateform (IUCT) for slides scanning, Fanny Grimal (IUCT) for microarrays hybridization, Laetitia Ligat (Pôle Technologique CRCT, U1037) for developing ImageJ macros for IHC quantification, the US006 for animal housing, and Lucie Fontaine (Histology Plateform, U1048). This work was supported by the Ligue Nationale Contre le Cancer (and Tarn, Gers and Haute-Garonne regional committees), the Labex TOUCAN, the Programme Hospitalo-Universitaire en Cancérologie CAPTOR, and the Institut National Du Cancer (2018-080).

DM was a recipient of a fellowship from LNCC and Fondation pour la Recherche Médicale (FDT20150532416); TGR, RB, and SS from the LNCC; and RS from the FRM (FDT40493).

Address correspondence to: Yvan Martineau and Stéphane Pyronnet, Cancer Research Center of Toulouse, 2 Avenue Hubert Curien, CS 53717, 31037 Toulouse Cedex 1, France. Phone: 33.5.82.74.17.38; Email: yvan.martineau@inserm.fr (YM). Phone: 33.5.82.74.16.54; Email: stephane.pyronnet@inserm.fr (SP).

1. Miller KD, et al. Cancer treatment and survivorship statistics, 2016. CA Cancer J Clin. 2016;66(4):271-289

2. Kleeff J, et al. Pancreatic cancer. Nat Rev Dis Primers. 2016;2:16022.

3. Royal RE, et al. Phase 2 trial of single agent Ipilimumab (anti-CTLA-4) for locally advanced or metastatic pancreatic adenocarcinoma. J Immunother. 2010;33(8):828-833

4. Cancer Genome Atlas Research Network. Electronic address: andrew_aguirre@dfci.harvard.edu, Cancer Genome Atlas Research Network. Integrated Genomic Characterization of Pancreatic Ductal Adenocarcinoma. Cancer Cell. 2017;32(2):185-203.e13.

5. Nicolle R, et al. Pancreatic Adenocarcinoma Therapeutic Targets Revealed by Tumor-Stroma Cross-Talk Analyses in Patient-Derived Xenografts. Cell Rep. 2017;21(9):2458-2470.

6. Piccirillo CA, Bjur E, Topisirovic I, Sonenberg N, Larsson O. Translational control of immune responses: from transcripts to translatomes. Nat Immunol. 2014;15(6):503-511.

7. Schwanhäusser B, et al. Global quantification of mammalian gene expression control. Nature. 2011;473(7347):337-342

8. Bhat M, Robichaud N, Hulea L, Sonenberg N, Pelletier J, Topisirovic I. Targeting the translation machinery in cancer. Nat Rev Drug Discov. 2015;14(4):261-278.

9. Hsieh AC, et al. The translational landscape of mTOR signalling steers cancer initiation and metastasis. Nature. 2012;485(7396):55-61

10. Larsson O, et al. Distinct perturbation of the translatome by the antidiabetic drug metformin. Proc Natl Acad Sci USA. 2012;109(23):8977-8982.

11. Andreev DE, et al. Translation of 5' leaders is pervasive in genes resistant to eIF2 repression. Elife. $2015 ; 4: \mathrm{e} 03971$.

12. Gandin V, et al. nanoCAGE reveals 5' UTR features that define specific modes of translation of functionally related MTOR-sensitive mRNAs. Genome Res. 2016;26(5):636-648.

13. Dowling RJ, et al. mTORC1-mediated cell proliferation, but not cell growth, controlled by the 4E-BPs. Science. 2010;328(5982):1172-1176.

14. Martineau Y, Azar R, Bousquet C, Pyronnet S. Anti-oncogenic potential of the eIF4E-binding proteins. Oncogene. 2013;32(6):671-677.

15. Thoreen CC, Chantranupong L, Keys HR, Wang T, Gray NS, Sabatini DM. A unifying model for mTORC1-mediated regulation of mRNA translation. Nature. 2012;485(7396):109-113.

16. Chio IIC, et al. NRF2 Promotes Tumor Maintenance by Modulating mRNA Translation in Pancreatic Cancer. Cell. 2016;166(4):963-976

17. Khalaileh A, et al. Phosphorylation of ribosomal protein S6 attenuates DNA damage and tumor suppression during development of pancreatic cancer. Cancer Res. 2013;73(6):1811-1820.

18. Martineau Y, et al. Pancreatic tumours escape from translational control through 4E-BP1 loss. Oncogene. 2014;33(11):1367-1374. 
19. Alain T, et al. eIF4E/4E-BP ratio predicts the efficacy of mTOR targeted therapies. Cancer Res. 2012;72(24):6468-6476.

20. Kim YY, et al. Eukaryotic initiation factor $4 \mathrm{E}$ binding protein family of proteins: sentinels at a translational control checkpoint in lung tumor defense. Cancer Res. 2009;69(21):8455-8462.

21. Azar R, Alard A, Susini C, Bousquet C, Pyronnet S. 4E-BP1 is a target of Smad4 essential for TGFbeta-mediated inhibition of cell proliferation. EMBO J. 2009;28(22):3514-3522.

22. Fragkos M, Ganier O, Coulombe P, Méchali M. DNA replication origin activation in space and time. Nat Rev Mol Cell Biol. 2015;16(6):360-374

23. Rogakou EP, Pilch DR, Orr AH, Ivanova VS, Bonner WM. DNA double-stranded breaks induce histone H2AX phosphorylation on serine 139. J Biol Chem. 1998;273(10):5858-5868.

24. Kiraly O, Gong G, Olipitz W, Muthupalani S, Engelward BP. Inflammation-induced cell proliferation potentiates DNA damage-induced mutations in vivo. PLoS Genet. 2015;11(2):e1004901.

25. Sans MD, DiMagno MJ, D'Alecy LG, Williams JA. Caerulein-induced acute pancreatitis inhibits protein synthesis through effects on eIF2B and eIF4F. Am J Physiol Gastrointest Liver Physiol. 2003;285(3):G517-G528.

26. Marion F, Kaloun EB, Lieby-Muller F, Perez M, Annereau J-P, Creancier L, inventors. Flavagline derivatives. US patent application 15/829,630. July 4, 2014.

27. Bordeleau ME, et al. Therapeutic suppression of translation initiation modulates chemosensitivity in a mouse lymphoma model. J Clin Invest. 2008;118(7):2651-2660.

28. Boussemart L, et al. eIF4F is a nexus of resistance to anti-BRAF and anti-MEK cancer therapies. Nature. 2014;513(7516):105-109.

29. Chu J, et al. CRISPR-Mediated Drug-Target Validation Reveals Selective Pharmacological Inhibition of the RNA Helicase, eIF4A. Cell Rep. 2016;15(11):2340-2347.

30. Galicia-Vázquez G, Cencic R, Robert F, Agenor AQ, Pelletier J. A cellular response linking eIF4AI activity to eIF4AII transcription. RNA. 2012;18(7):1373-1384

31. Duluc C, et al. Pharmacological targeting of the protein synthesis mTOR/4E-BP1 pathway in cancer-associated fibroblasts abrogates pancreatic tumour chemoresistance. EMBO Mol Med. 2015;7(6):735-753.

32. Waddell N, et al. Whole genomes redefine the mutational landscape of pancreatic cancer. Nature. 2015;518(7540):495-501.

33. Wolfe AL, et al. RNA G-quadruplexes cause eIF4A-dependent oncogene translation in cancer. Nature. 2014;513(7516):65-70.

34. Kikin O, D'Antonio L, Bagga PS. QGRS Mapper: a web-based server for predicting G-quadruplexes in nucleotide sequences. Nucleic Acids Res. 2006;34(Web Server issue):W676-W682.

35. Waldron JA, Raza F, Le Quesne J. eIF4A alleviates the translational repression mediated by classical secondary structures more than by G-quadruplexes. Nucleic Acids Res. 2018;46(6):3075-3087.

36. Cencic R, et al. Antitumor activity and mechanism of action of the cyclopenta[b]benzofuran, silvestrol. PLoS ONE. 2009;4(4):e5223.

37. Olive KP, et al. Inhibition of Hedgehog signaling enhances delivery of chemotherapy in a mouse model of pancreatic cancer. Science. 2009;324(5933):1457-1461.

38. Forbes SA, et al. COSMIC: somatic cancer genetics at high-resolution. Nucleic Acids Res. 2017;45(D1):D777-D783.

39. Cendrowski J, et al. Mnk1 is a novel acinar cell-specific kinase required for exocrine pancreatic secretion and response to pancreatitis in mice. Gut. 2015;64(6):937-947.

40. Furic L, et al. eIF4E phosphorylation promotes tumorigenesis and is associated with prostate cancer progression. Proc Natl Acad Sci USA. 2010;107(32):14134-14139.

41. Robichaud N, et al. Phosphorylation of eIF4E promotes EMT and metastasis via translational control of SNAIL and MMP-3 Oncogene. 2015;34(16):2032-2042.

42. Muller D, et al. 4E-BP restrains eIF4E phosphorylation. Translation (Austin). 2013;1(2):e25819.

43. Thompson PA, et al. Preclinical Evaluation of eFT226, a Novel, Potent and Selective eIF4A Inhibitor with Anti-Tumor Activity in B-Cell Malignancies. Blood. 2017;130(Suppl 1):1530.

44. Tsukumo Y, Alain T, Fonseca BD, Nadon R, Sonenberg N. Translation control during prolonged mTORC1 inhibition mediated by 4E-BP3. Nat Commun. 2016;7:11776.

45. Petroulakis E, et al. p53-dependent translational control of senescence and transformation via 4E-BPs. Cancer Cell. 2009;16(5):439-446. 